\title{
Die Grundlage der allgemeinen Relativitätstheorie
}

von

A. Einstein

$\checkmark$

Leipzig :: Verlag von Johann Ambrosius Barth :: 1916 
Sonderdruck aus den „Annalen der Physik"

Band 49, 1916

Inhalt.

Einleitung . . . . . . . . . . 5

A. Prinzipielle Erwägungen zum Postulat der Relativität

$\S$ 1. Bemerkungen zu der speziellen Relativitätstheorie. . 7

\& 2. Über die Gründe, welche eine Erweiterung des Relativitätspostulates nahelegen

\& 3. Das Raum-Zeit-Kontinuum. Forderung der allgemeinen Kovarianz für die die allgemeinen Naturgesetze ausdrüekenden Gleichungen

\& 4. Beziehung der vier Koordinaten zu räumlichen und zeitlichen MéBergebnissen. Analytischer Ausdruck für

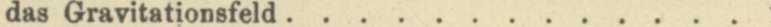

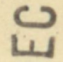
$\square$ B. Mathematische Hilfsmittel für die Aufstellung allgemein kovarianter Gleichungen

5. 5. Kontravarianter und kovarianter Vierervektor * "

5. Tentavarianter und kovarianter

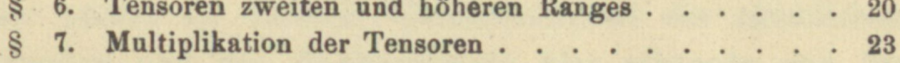

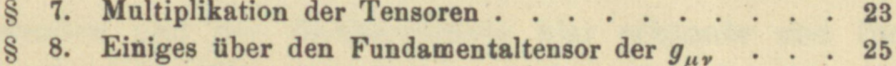

$\S$ 9. Gleichung der geodätischen Linie (bzw. der Punktbewegung)

Bildung von Tensoren durch Differentiation . 30

\$ 11. Einige Spezialfălle von besonderer Bedeutung . . . 34

\$ 12. Der Riemann-Christoffelsche Tensor. . . . . . 38

C. Theorie des Gravitationsfeldes. . . . . . . . . . . . 41

\$ 13. Bewegungsgleichung des materiellen Punktes im Gravitationsfeld. Ausdruck für die Feldkomponenten der Gravitation

\& 14. Die Feldgleichungen der Gravitation bei Abwesenheit von Materie. . . . . . . . . . .

\$ 15. Hamiltonsche Funktion für das Gravitationsfeld, Impulsenergiesatz . . . . . . . . . . . . 
8 16. AllgemeineFassung der Feldgleichungen der Gravitation Seite

\$ 17. Die Erhaltungssätze im allgemeinen Falle. . . . . 48

§ 18. Der Impulsenergiesatz für die Materie als Folge der Feldgleichungen ......... 49

D. Die "materiellen" Vorgänge . . . . . . . . . . . . . . . 51

§ 19. Eulersche Gleichungen für reibungslose adiabatische Flüssigkeiten ............ . 51

8 20. Maxwellsche elektromagnetische Feldgleichungen für das Vakuum . . . . . . . . . . . . . . 53

E. \$ 21. Newtons Theorie als erste Näherung . . . . . . 57

\& 22. Verhalten von MaBstäben und Uhren im statischen Gravitationsfelde. Krümmung der Lichtstrahlen. Perihelbewegung der Planetenbahnen . . . . . . 60

Einleitung.

Die im nachfolgenden dargelegte Theorie bildet die denkbar weitgehendste Verallgemeinerung der heute allgemein als „Relativitätstheorie“ bezeichneten Theorie; die letztere nenne ich im folgenden zur Unterscheidung von der ersteren ,spezielle Relativitätstheorie" und setze sie als bekannt voraus. Die Verallgemeinerung der Relativitätstheorie wurde sehr erleichtert durch die Gestalt, welche der speziellen Relativitätstheorie durch Minkowski gegeben wurde, welcher Mathematiker zuerst die formale Gleichwertigkeit der räumlichen Koordinaten und der Zeitkoordinate klar erkannte und für den Aufbau der Theorie nutzbar machte. Die für die allgemeine Relativitätstheorie nötigen mathematischen Hilfsmittel lagen fertig bereit in dem ,absoluten Differentialkalkül“, welcher auf den Forschungen von Gauss, Riemann und Christoffel über nichteuklidische Mannigfaltigkeiten ruht und von Ricci und Levi-Civita in ein System gebracht und bereits auf Probleme der theoretischen Physik angewendet wurde. Ich habe im Abschnitt B der vorliegenden Abhandlung alle für uns nötigen, bei dem Physiker nicht als bekannt vorauszusetzenden mathematischen Hilfsmittel in möglichst einfacher und durchsichtiger Weise entwickelt, so daB or 
Studium mathematischer Literatur für das Verständnis der vorliegenden Abhandlung nicht erforderlich ist. Endlich sei an dieser Stelle dankbar meines Freundes, des Mathematikers Grossmann, gedacht, der mir durch seine Hilfe nicht nur das Studium der einschlägigen mathematischen Literatur ersparte, sondern mich auch beim Suchen nach den Feldgleichungen der Gravitation unterstützte.

\section{A. Prinzipielle Erwägungen zum Postulat der Relativitåt.}

§ 1. Bemerkungen zu der speziellen Relativitätstheorie.

Der speziellen Relativitätstheorie liegt folgendes Postulat zugrunde, welchem auch durch die Galilei-Newtonsche Mechanik Genüge geleistet wird: Wird ein Koordinatensystem $K$ so gewählt, daß in bezug auf dasselbe die physikalischen Geso gewählt, daß in ihrer einfachsten Form gelten, so gelten dieselben Gesetze auch in bezug auf jedes andere Koordinatensystem $K^{\prime}$, das relativ zu $K$ in gleichförmiger Translationsbewegung begriffen ist. Dieses Postulat nennen wir ,spezielles Relativitätsprinzip“. Durch das Wort „speziell“ soll angedeutet werden, daß das Prinzip auf den Fall beschränkt ist, daß $K^{\prime}$ eine gleichförmige Translationsbewegung gegen $K$ ausführt, daß sich aber die Gleichwertigkeit von $K^{\prime}$ und $K$ nicht auf den Fal ungleichförmiger Bewegung von $K^{\prime}$ gegen $K$ erstreckt.

Die spezielle Relativitätstheorie weicht also von der klassischen Mechanik nicht durch das Relativitätspostulat ab, sondern allein durch das Postulat von der Konstanz der Vakuum-Lichtgeschwindigkeit, aus welchem im Verein mit dem speziellen Relativitätsprinzip die Relativität der Gleichzeitigkejt sowie die Lorentztransformation und die mit dieser verknüpften Gesetze über das Verhalten bewegter starrer Körper und Uhren in bekannter Weise folgen.

Die Modifikation, welche die Theorie von Raum und Zeit durch die spezielle Relativitätstheorie erfahren hat, ist zwar eine tiefgehende; aber ein wichtiger Punkt blieb unangetastet Auch gemäß der speziellen Relativitätstheorie sind nämlich die Sätze der Geometrie unmittelbar als die Gesetze über die möglichen relativen Lagen (ruhender) fester Körper zu deuten, allgemeiner die Sätze der Kinematik als Sätze, welche das Verhalten von Meßkörpern und Uhren beschreiben. Zwei 
hervorgehobenen materiellen Punkten eines ruhenden (starren) Körpers entspricht hierbei stets eine Strecke von ganz be stimmter Länge, unabhängig von Ort und Orientierung des Körpers sowie von der Zeit; zwei hervorgehobenen Zeigerstellungen einer relativ zum (berechtigten) Bezugssystem ruhenden Uhr entspricht stets eine Zeitstrecke von bestimmter Länge, unabhängig von Ort und Zeit. Es wird sich bald zeigen, daß die allgemeine Relativitätstheorie an dieser einfachen physikalischen Deutung von Raum und Zeit nicht festhalten kann.

\section{2. Uber die Gründe, welche eine Erweiterung des Relativitäts-} postulates nahelegen.

Der klassischen Mechanik und nicht minder der speziellen Relativitätstheorie haftet ein erkenntnistheoretischer Mangel an, der vielleicht zum ersten Male von E. Mach klar hervorgehoben wurde. Wir erläutern ihn am folgenden Beispiel. Zwei flüssige Körper von gleicher Größe und Art schweben frei im Raume in so großer Entfernung voneinander (und von allen übrigen Massen), daß nur diejenigen Gravitationskräfte berücksichtigt werden müssen, welche die Teile eines dieser Körper aufeinander ausüben. Die Entfernung der Körper voneinander sei unveränderlich. Relative Bewegungen der Teile eines der Körper gegeneinander sollen nicht auftreten. Aber jede Masse soll - von einem relativ zu der anderen Masse ruhenden Beobachter aus beurteilt - um die Verbindungslinie der Massen mit konstanter Winkelgeschwindigkeit rotieren (es ist dies eine konstatierbare Relativbewegung beider Massen). Nun denken wir uns die Oberflächen beider Körper $\left(S_{1}\right.$ und $\left.S_{2}\right)$ mit Hilfe (relativ ruhender) Maßstäbe ausgemessen; es ergebe sich, daß die Oberfläche von $S_{1}$ eine Kugel, die von $S_{2}$ ein Rotationsellipsoid sei.

Wir fragen nun: Aus welchem Grunde verhalten sich die Körper $S_{1}$ und $S_{2}$ verschieden? Eine Antwort auf diese Frage kann nur dann als erkenntnistheoretisch befriedigend ${ }^{1}$ ) anerkannt werden, wenn die als Grund angegebene Sache eine beobachtbare Erfahrungstatsache ist; denn das Kausalitäts-

1) Eine derartige erkenntnistheoretisch befriedigende Antwort kann natürlich immer noch physikalisch unzutreffend sein, falls sie mit anderen Erfahrungen im Widerspruch ist. gesetz hat nur dann den Sinn einer Aussage über die Erfahrungswelt, wenn als Ursachen und Wirkungen letzten Endes nur beobachtbare Tatsachen auftreten.

Die Newtonsche Mechanik gibt auf diese Frage keine befriedigende Antwort. Sie sagt nämlich folgendes. Die $\mathrm{Ge}$ setze der Mechanik gelten wohl für einen Raum $R_{1}$, gegen welchen der Körper $S_{1}$ in Ruhe ist, nicht aber gegenüber einem Raume $R_{2}$, gegen welchen $S_{2}$ in Ruhe ist. Der berechtigte Galileische Raum $R_{1}$, der hierbei eingeführt wird (bzw. die Relativbewegung $\mathrm{zu}$ ihm), ist aber eine blo $\beta$ fingierte Ursache, keine beobachtbare Sache. Fs ist also klar, daß die Newtonsche Mechanik der Forderung der Kausalität in dem betracbteten Falle nicht wirklich, sondern nur scheinbar Genüge leistet, indem sie die bloß fingierte Ursache $R_{1}$ für das beobachtbare verschiedene Verbalten der Körper $S_{1}$ und $S_{2}$ verantwortlich macht.

Eine befriedigende Antwort auf die oben aufgeworfene Frage kann nur so lauten: Das aus $S_{1}$ und $S_{2}$ bestehende physikalische System zeigt für sich allein keine denkbare Ursache, auf welche das verschiedene Verhalten von $S_{1}$ und $S_{2}$ zurückgeführt werden könnte. Die Ursache muß also außer halb dieses Systems liegen. Man gelangt zu der Auffassung, $\mathrm{da} B$ die allgemeinen Bewegungsgesetze, welche im speziellen die Gestalten von $S_{1}$ und $S_{2}$ bestimmen, derart sein müssen, da $B$ das mechanische Verhalten von $S_{1}$ und $S_{2}$ ganz wesentlich durch ferne Massen mitbedingt werden muß, welche wir nicht $\mathrm{zu}$ dem betrachteten System gerechnet hatten. Diese fernen Massen (und ihre Relativbewegungen gegen die betrachteten Körper) sind dann als Träger prinzipiell beobachtbarer Ursachen für das verschiedene Verhalten unserer betrachteten Körper anzusehen; sie übernehmen die Rolle der fingierten Ursache $R_{1}$. Von allen denkbaren, relativ zueinander beliebig bewegten Räumen $R_{1}, R_{2}$ usw. darf a priori keiner als bevorzugt angesehen werden, wenn nicht der dargelegte erkenntnistheoretische Einwand wieder aufleben soll. Die Gesetze der Physit müssen so beschaffen sein, daß sie in bezug auf beliebig bewegte Bezugssysteme gelten. Wir gelangen also auf diesem Wege zu einer Erweiterung des Relativitätspostulates.

Außer diesem schwerwiegenden erkenntnistheoretischen Argument spricht aber auch eine wohlbekannte physikalische 
Tatsache für eine Erweiterung der Relativitätstheorie. Es sei $K$ ein Galileisches Bezugssystem, d. h. ein solches, relativ $\mathrm{zu}$ welchem (mindestens in dem betrachteten vierdimensionalen Gebiete) eine von anderen hinlänglich entfernte Masse sich geradlinig und gleicbförmig bewegt. Es sei $K^{\prime}$ ein ziweites Koordinatensystem, welches relativ zu $K$ in gleichförmig beschleunigter Translationsbewegung sei. Relativ zu $K^{\prime}$ führte dann eine von anderen hinreichend getrennte Masse eine beschleunigte Bewegung aus, derart, da $\beta$ deren Beschleunigung und Beschleunigungsrichtung von ihrer stofflichen Zusammensetzung und ihrem physikalischen Zustande unabhängig ist.

Kann ein relativ zu $K^{\prime}$ ruhender Beobachter hieraus den Schluß ziehen, daß er sich auf einem ,wirklich“ beschleunigten Bezugssystem befindet? Diese Frage ist zu verneinen; denn das vorhin genannte Verhalten frei beweglicher Massen relativ zu $K^{\prime}$ kann ebensogut auf folgende Weise gedeutet werden. Das Bezugssystem $K^{\prime}$ ist unbeschleunigt; in dem betrachteten zeiträumlichen Gebiete herrscht aber ein Gravitationsfeld, welches die beschleunigte Bewegung der Körper relativ zu $K^{\prime}$ erzeugt.

Diese Auffassung wird dadurch ermöglicht, daß uns die Erfahrung die Existenz eines Kraftfeldes (nämlich des Gravitationsfeldes) gelehrt hat, welches die merkwürdige Eigenschaft hat, allen Körpein dieselbe Beschleunigung zu erteilen. ${ }^{1}$ ) Das mechanische Verhalten der Körper relativ zu $K^{\prime}$ ist dasselbe, wie es gegenüber Systemen sich der Erfahrung darbietet, die wir als ,ruhende" bzw. als ",berechtigte" Systeme anzusehen gewohnt sind; deshalb liegt es auch vom physikalischen Standpunkt nahe, anzunehmen, daß die Systeme $K$ und $K^{\prime}$ beide mit demselben Recht als ,ruhend " angesehen werden können, bzw. daß sie als Bezugssysteme für die physikalische Beschreibung der Vorgänge gleichberechtigt seien.

Aus diesen Erwägungen sieht man, daß die Durchführung der allgemeinen Relativitätstheorie zugleich zu einer Theorie der Gravitation führen muß; denn man kann ein Gravitationsfeld durch bloße Änderung des Koordinatensystems ,erzeugen“. Ebenso sieht man unmittelbar, daß das Prinzip von der Kon-

1) $\mathrm{Da}$ das Gravitationsfeld diese Eigenschaft mit großer Genauigkeit besitzt, hat Eötvös experimentell bewiesen. stanz der Vakuum-Lichtgeschwindigkeit eine Modifikation erfahren muß. Denn man erkennt leicht, daß die Bahn eines Lichtstrahles in bezug auf $K^{\prime}$ im allgemeinen eine krumme sein muß, wenn sich das Licht in bezug auf $K$ geradlinig und mit bestimmter, konstanter Geschwindigkeit fortpflanzt.

8 3. Das Raum-Zeit-Kontinuum. Forderung der allgemeinen Kovarianz für die die allgemeinen Naturgesetze ausdrückenden Gleichungen.

In der klassischen Mechanik sowie in der speziellen Relativitätstheorie haben die Koordinaten des Raumes und der Zeit eine unmittelbare physikalische Bedeutung. Ein Punktereignis hat die $X_{1}$-Koordinate $x_{1}$, bedeutet: Die nach den Regeln der Euklidischen Geometrie mittels starrer Stäbe ermittelte Projektion des Punktereignisses auf die $X_{1}$-Achse wird erhalten, indem man einen bestimmten Stab, den Einheitsmaßstab, $x_{1}$ mal vom Anfangspunkt des Koordinatenkörpers auf der (positiven) $X_{1}$-Achse abträgt. Ein Punkt hat die $X_{4}$-Koordinate $x_{4}=t$, bedeutet: Eine relativ zum Koordinatensystem ruhend angeordnete, mit dem Punktereignis räumlich (praktisch) zusammenfallende Einheitsubr, welche nach bestimmten Vorschriften gerichtet ist, bat $x_{4}=t$ Perioden zurückgelegt beim Eintreten des Punktereignisses. ${ }^{1}$ )

Diese Auffassung von Raum und Zeit schwebte den Physikern stets, wenn auch meist unbewußt, vor, wie aus der Rolle klar erkennbar jst, welche diese Begriffe in der messenden Physik spielen; diese Auffassung mußte der Leser auch der zweiten Betrachtung des letzten Paragraphen zugrunde legen, um mit diesen Ausführungen einen Sinn verbinden zu können. Aber wir wollen nun zeigen, daß man sie fallen lassen und durch eine allgemeinere ersetzen $m u ß$, um das Postulat der allgemeinen Relativität durchführen $\mathrm{zu}$ können, falls die spezielle Relativitätstheorie für den Grenzfall des Fehlens eines Gravitationsfeldes zutrifft.

1) Die Konstatierbarkeit der „Gleichzeitigkeit” für räumlich unmittelbar benachbarte Ereignisse, oder - präziser gesagt - für das raumzeitliche unmittelbare Benachbartsein (Koinzidenz) nehmen wir an, ohne für diesen fundamentalen Begriff eine Definition zu geben. 
Wir fübren in einem Raume, der frei sei von Gravitationsfeldern, ein Galileisches Bezugssystem $K(x, y, z, t)$ ein, und außerdem ein relativ zu $K$ gleichförmig rotierendes Koordinatensystem $K^{\prime}\left(x^{\prime}, y^{\prime}, z^{\prime} t^{\prime}\right)$. Die Anfangspunkte beider Systeme sowie deren Z-Achsen mögen dauernd zusammenfallen. Wir wollen zeigen, daß für eine Raum-Zeitmessung im System $K^{\prime}$ die obige Festsetzung für die physikalische Bedeutung von Längen und Zeiten nicht aufrecht erhalten werden kann. Aus Symmetriegründen ist klar, daß ein Kreis um den Anfangspunkt in der $X-Y$-Ebene von $K$ zugleich als Kreis in der $X^{\prime}-Y^{\prime}$-Ebene von $K^{\prime}$ aufgefaßt werden kann. Wir denken uns nun Umfang und Durchmesser dieses Kreises mit einem (relativ zum Radius unendlich kleinen) Einheitsmaßstabe ausgemessen und den Quotienten beider Meßresultate gebildet. Würde man dieses Experiment mit einem relativ zum Galileischen System $K$ ruhenden Maßstabe ausführen, so würde man als Quotienten die Zahl $\pi$ erhalten. Das Resultat der mit einem relativ zu $K^{\prime}$ ruhenden Maßstabe ausgeführten Bestimmung würde eine Zahl sein, die größer ist als $\pi$. Man erkennt dies leicht, wenn man den ganzen Meßproze $B$ vom, ,ruhenden“ System $K$ aus beurteilt und berücksichtigt, daß der peripherisch angelegte $\mathrm{Maßstab}$ eine Lorentzverkürzung erleidet, der radial angelegte Maßstab aber nicht. Es gilt daher in bezug auf $K^{\prime}$ nicht die Euklidische Geometrie; der oben festgelegte Koordinatenbegriff, welcher die Gültigkeit der Euklidischen Geometrie voraussetzt, versagt also mit Bezug auf das System $K^{\prime}$. Ebensowenig kann man in $K^{\prime}$ eine den physikalischen Bedürfnissen entsprechende Zeit einführen, welche durch relativ zu $K^{\prime}$ ruhende, gleich beschaffene Uhren angezeigt wird. Um dies einzusehen, denke man sich im Koordinatenursprung und an der Peripherie des Kreises je eine von zwei gleich beschaffenen Uhren angeordnet und vom ,ruhenden" System $K$ aus betrachtet. Nach einem bekannten Resultat der speziellen Relativitätstheorie geht - von $K$ aus beurteilt - die auf der Kreisperipherie angeordnete Uhr langsamer als die im Anfangspunkt angeordnete Uhr, weil erstere Uhr bewegt ist letztere aber nicht. Ein im gemeinsamen Koordinatenursprung befindlicher Beobachter, welcher auch die an der Peripherie befindliche Uhr mittels des Lichtes zu beobachten fähig wäre, würde also die an der Peripherie angeordnete Uhr langsamer gehen sehen als die neben ihm angeordnete Uhr. Da er sich nicht dazu entschließen wird, die Lichtgeschwindigkeit auf dem in Betracht kommenden Wege explizite von det Zeit abhängen zu lassen, wird er seine Beobachtung dahin interpretieren, daß die Uhr an der Peripherie ,wirklich" langsamer gehe als die im Ursprung angeordnete. Er wird also nicht umhin können, die Zeit so zu definieren, daß die Ganggeschwindigkeit einer Uhr vom Orte abhängt.

Wir gelangen also zu dem Ergebnis: In der allgemeinen Relativitätstheorie können Raum- und Zeitgrößen nicht so definiert werden, daß räumliche Koordinatendifferenzen unmittelbar mit dem Einheitsmaßstab, zeitliche mit einer Normaluhr gemessen werden könnten.

Das bisherige Mittel; in das zeiträumliche Kontinuum in bestimmter Weise Koordinaten zu legen, versagt also, und es scheint sich auch kein anderer Wfg darzubieten, der gestatten würde, der vierdimensionalen Welt Koordinatensysteme so anzupassen, daß bei ihrer Verwendung eine besonders einfache Formulierung der Naturgesetze zu erwarten wäre. Es bleibt daher nichts anderes übrig, als alle denkbaren ${ }^{1}$ ) Koordinatensysteme als für die Naturbeschreibung prinzipiell gleichberechtigt anzusehen. Dies kommt auf die Forderung hinaus:

Die allgemeinen Naturgesetze sind durch Gleichungen auszudrücken, die für alle Koordinatensysteme gelten, $d . h$. die beliebigen Substitutionen gegenüber kovariant (allgemein kovariant) sind.

Es ist klar, daß eine Physik, welche diesem Postulat genügt, dem allgemeinen Relativitätspostulat gerecht wird. Denn in allen Substitutionen sind jedenfalls auch diejenigen enthalten, welche allen Relativbewegungen der (dreidimensionalen) Koordinatensysteme entsprechen. Daß diese Forderung der allgemeinen Kovarianz, welche dem Raum und der Zeit den letzten Rest physikalischer Gegenständlichkeit nehmen, eine natürliche Forderung ist, geht aus folgender Überlegung

1) Von gewissen Beschränkungen, welche der Forderung der eindeutigen Zuordnung und derjenigen der Stetigkeit entsprechen, wollen wir hier nicht sprechen. 
hervor. Alle unsere zeiträumlichen Konstatierungen laufen stets auf die Bestimmung zeiträumlicher Koinzidenzen hinaus. Bestände beispielsweise das Geschehen nur in der Bewegung materieller Punkte, so wäre letzten Endes nichts beobachtbar als die Begegnungen zweier oder mebrerer dieser Punkte. Auch die Ergebnisse unserer Messungen sind nichts anderes als die Konstatierung derartiger Begegnungen materieller Punkte unserer Maßstäbe mit anderen materiellen Punkten bzw. Koinzidenzen zwischen Uhrzeigern, Zifferblattpunkten und ins Auge gefaßten, am gleichen Orte und zur gleichen Zeit stattfindenden Punktereignissen.

Die Einführung eines Bezugssystems dient zu nichts anderem als zur leichteren Beschreibung der Gesamtheit solcher Koinzidenzen. Man ordnet der Welt vier zeiträumliche Variable $x_{1}, x_{2}, x_{3}, x_{4}$ zu, derart, daß jedem Punktereignis ein Wertesystem der Variablen $x_{1} \ldots x_{4}$ entspricht. Zwei koinzidierenden Punktereignissen entspricht dasselbe Wertesystem der Variablen $x_{1} \ldots x_{4} ; \mathrm{d} . \mathrm{h}$. die Koinzidenz ist durch die Übereinstimmung der Koordinaten charakterisiert. Führt man statt der Variablen $x_{1} \ldots x_{4}$ beliebige Funktionen derselben, $x_{1}{ }^{\prime}, x_{2}{ }^{\prime}, x_{3}{ }^{\prime}, x_{4}{ }^{\prime}$ als neues Koordinatensystem ein, so da $\beta$ die Wertesysteme einander eindentig zugeordnet sind, so ist die Gleichheit aller vier Koordinaten auch im neuen System der Ausdruck für die raumzeitliche Koinzidenz zweier Punktereignisse. Da sich alle unsere physikalischen Erfahrungen letzten Endes auf solche Koinzidenzen zurückführen lassen, ist zunächst kein Grund vorhanden, gewisse Koordinatensysteme vor anderen zu bevorzugen, d. h. wir gelangen zu der Forderung der allgemeinen Kovarianz.

\section{\$ 4. Beziehung der vier Koordinaten zu räumlichen und zeit- lichen Meßergebnissen.}

Analytischer Ausdruck für das Gravitationsfeld.

Es kommt mir in dieser Abhandlung nicht darauf an, die allgemeine Relativitätstheorie als ein möglichst einfaches logisches System mit einem Minimum von Axiomen darzustellen. Sondern es ist mein Hauptziel, diese Theorie so zu entwickeln, daß der Leser die psychologische Natürlichkeit des eingeschlagenen Weges empfindet und daß die zugrunde gelegten Voraussetzungen durch die Erfahrung möglichst gesichert erscheinen. In diesem Sinne sei nun die Voraussetzung eingeführt:

Für unendlich kleine vierdimensionale Gebiete ist die Relativitätstheorie im engeren Sinne bei passender Koordinatenwahl zutreffend.

Der Beschleunigungszustand des unendlich kleinen (,örtlichen") Koordinatensystems ist hierbei so zu wählen, daß ein Gravitationsfeld nicht auftritt; dies ist für ein unendlich kleines Gebiet möglich. $X_{1}, X_{2}, X_{3}$ seien die räumlichen Koordinaten; $X_{4}$ die zugehörige, in geeignetem Maßstabe gemessene $^{1}$ ) Zeitkoordinate. Diese Koordinaten haben, wenn ein starres Stäbchen als Einheitsmaßstab gegeben gedacht wird, bei gegebener Orientierung des Koordinatensystems eine unmittelbare physikalische Bedeutung im Sinne der speziellen Relativitätstheorie. Der Ausdruck

(1) $\quad d s^{2}=-d X_{1}{ }^{2}-d X_{2}{ }^{2}-d X_{3}{ }^{2}+d X_{4}{ }^{2}$

hat dann nach der speziellen Relativitätstheorie einen von der Orientierung des lokalen Koordinatensystems unabhängigen, durch Raum-Zeitmessung ermittelbaren Wert. Wir nennen $d s$ die Größe des zu den unendlich benachbarten Punkten des vierdimensionalen Raumes gehörigen Linienelementes. Ist das zu dem Element $\left(d X_{1} \ldots d X_{4}\right)$ gehörige $d s^{2}$ positiv, so nennen wir mit Min kowski ersteres zeitartig, im entgegengesetzten Falle raumartig.

Zu dem betrachteten „Linienelement“" bzw. zu den beiden unendlich benachbarten Punktereignissen gehören auch bestimmte Differentiale $d x_{1} \ldots d x_{4}$ der vierdimensionalen Koordinaten des gewählten Bezugssystems. Ist dieses sowie ein „lokales“ System obiger Art für die betrachtete Stelle gegeben, so werden sich hier die $d X_{v}$ durch bestimmte lineare homogene Ausdrücke der $d x_{\sigma}$ darstellen lassen:

$$
d X_{\nu}=\sum_{\sigma} \mu_{\nu \sigma} d x_{\sigma} .
$$

Setzt man diese Ausdrücke in (1) ein, so erhält man

$$
d s^{2}=\sum_{\sigma \tau} g_{\sigma \tau} d x_{\sigma} d x_{\tau} .
$$

1) Die Zeiteinheit ist so zu wählen, da ß die Vakuum-Lichtgeschwindigkeit - in dem „lokalen“ Koordinatensystem gemessen - gleich 1 wird. 
wobei die $g_{a \tau}$ Funktionen der $x_{\sigma}$ sein werden, die nicht mehr von der Orientierung und dem Bewegungszustand des ,lokalen“ Koordinatensystems abhängen können; denn $d s^{2}$ ist eine durch Maßstab-Uhrenmessung ermittelbare, zu den betrachteten, zeiträumlich unendlich benachbarten Punktereignissen gehörige, unabhängig von jeder besonderen Koordinatenwahl definierte Größe. Die $g_{\sigma r}$ sind hierbei so zu wählen, daß $g_{o \tau}=g_{\tau \sigma}$ ist; die Summation ist über alle Werte von $\sigma$ und $\tau$ zu erstrecken, so da $\beta$ die Summe aus $4 \times 4$ Summanden besteht, von denen 12 paarweise gleich sind.

Der Fall der gewöhnlichen Relativitätstheorie geht aus dem hier Betrachteten hervor, falls es, vermöge des besonderen Verhaltens der $g_{\sigma \tau}$ in einem endlichen Gebiete, möglich ist, in diesem das Bezugssystem so zu wählen, daß die $g_{\sigma \tau}$ die konstanten Werte

$$
\left\{\begin{array}{rrrr}
-1 & 0 & 0 & 0 \\
0 & -1 & 0 & 0 \\
0 & 0 & -1 & 0 \\
0 & 0 & 0 & +1
\end{array}\right.
$$

annehmen. Wir werden später sehen, daß die Wahl solcher Koordinaten für endliche Gebiete im allgemeinen nicht möglich ist.

Aus den Betrachtungen der $\S \S 2$ und 3 geht hervor, daß die Größen $g_{\sigma \tau}$ vom physikalischen Standpunkte aus als diejenigen Größen anzusehen sind, welche das Gravitationsfeld in bezug auf das gewählte Bezugssystem beschreiben. Nehmen wir nämlich zunächst an, es sei für ein gewisses betrachtetes vierdimensionales Gebiet bei geeigneter Wahl der Koordinaten die spezielle Relativitätstheorie gültig. Die $g_{\sigma r}$ haben dann die in (4) angegebenen Werte. Ein freier materieller Punkt bewegt sich dann bezüglich dieses Systems geradlinig gleichförmig. Führt man nun durch eine beliebige Substitution neve Raum-Zeitkoordinaten $x_{1} \ldots x_{4}$ ein, so werden in diesem neuen System die $g_{\mu \nu}$ nicht mehr Konstante, sondern Raum-Zeitfunktionen sein. Gleichzeitig wird sich die $\mathrm{Be}$ wegung des freien Massenpunktes in den neuen Koordinaten als eine krummlinige, nicht gleichförmige, darstellen, wobei dies Bewegungsgesetz unabhängig sein wird von der Natur des bewegten Massenpunktes. Wir werden also diese $\mathrm{Be}$ - wegung als eine solche unter dem Einfluß eines Gravitationsfeldes deuten. Wir sehen das Auftreten eines Gravitationsfeldes geknüpft an eine raumzeitliche Veränderlichkeit der $g_{\sigma_{r}}$ Auch in dem allgemeinen Falle, daß wir nicht in einem endlichen Gebiete bei passender Koordinatenwahl die Gültigkeit der speziellen Relativitätstheorie herbeiführen können, werden wir an der Auffassung festzuhalten haben, daß die $g_{o \tau}$ das Gravitationsfeld beschreiben.

Die Gravitation spielt also gemä $B$ der allgemeinen Relativitätstheorie eine Ausnahmerolle gegenüber den übrigen, insbesondere den elektromagnetischen Kräften, indem die das Gravitationsfeld darstellenden 10 Funktionen $g_{\sigma \tau}$ zugleich die metrischen Eigenschaften des vierdimensionalen Meßraumes bestimmen. 


\section{\$ 5. Kontravarianter und kovarianter Vierervektor.}

Kontravarianter Vierervektor. Das Linienelement ist definiert durch die vier „Komponenten“ $d x_{v}$, deren Transformationsgesetz durch die Gleichung

$$
d x_{\sigma}^{\prime}=\sum_{v} \frac{\partial x_{g}^{\prime}}{\partial x_{v}} d x_{v}
$$

B. Mathematisehe Hilfsmittel fïr die Aufstellung allgemein kovarianter Gleichungen.

Nachdem wir im vorigen gesehen haben, daß das allgemeine Relativitätspostulat zu der Forderung führt, daß die Gleichungssysteme der Physik beliebigen Substitutionen der Koordinaten $x_{1} \ldots x_{4}$ gegenüber kovariant sein müssen, haben wir zu überlegen, wie derartige allgemein kovariante Gleichungen gewonnen werden können. Dieser rein mathematischen Aufgabe wenden wir uns jetzt zu; es wird sich dabei zeigen, daß bei deren Lösung die in Gleichung (3) angegebene Invariante $d s$ eine fundamentale Rolle spielt, welche wir in Anlehnung an die Gausssche Flächentheorie als „LinienHement" bezeichnet haben.

Der Grundgedanke dieser allgemeinen Kovariantentheorie ist folgender. Es seien gewisse Dinge (,Tensoren“) mit Bezùg uf jedes Koordinatensystem definiert durch eine Anzahl laumfunktionen, welche die ,Komponenten“ des Tensors unannt werden. Es gibt dann gewisse Regeln, nach welchen diese Komponenten für ein neues Koordinatensystem berechnet werden, wenn sie für das ursprüngliche System bekannt sind, und wenn die beide Systeme verknüpfende Transformation bekannt ist. Die nachher als Tensoren bezeichneten Dinge sind ferner dadurch gekennzeichnet, daß die Transformationsgleichungen für ihre Komponenten linear und homogen sind. Demnach verschwinden sämtliche Komponenten im neuen System, wenn sie im ursprünglichen System sämtlich verschwinden. Wird also ein Naturgesetz durch das Nullsetzen aller Komponenten eines Tensors formuliert, so ist es allgemein kovariant; indem wir die Bildungsgesetze der Tensoren untersuchen, erlangen wir die Mittel zur Aufstellung allgemein kovarianter Gesetze. ausgedrückt wird. Die $d x_{\sigma}^{\prime}$ drücken sich linear und homogen durch die $d x$, aus; wir können diese Koordinatendifferentiale $d x$ daher als die Komponenten eines "Tensors" ansehen, den wir speziell als kontravarianten Vierervektor bezeichnen. Jedes Ding, was bezüglich des Koordinatensystems durch vier Größen $A^{v}$ definiert ist, die sich nach demselben Gesetz

$$
A^{\sigma^{\prime}}=\sum_{v} \frac{\partial x_{\sigma}^{\prime}}{\partial x_{\nu}} A^{v}
$$

transformieren, bezeichnen wir ebenfalls als kontravarianten Vierervektor. Aus (5a) folgt sogleich, da $B$ die Summen $\left(A^{\sigma} \pm B^{\sigma}\right)$ ebenfalls Komponenten eines Vierervektors sind, wenn $A^{\sigma}$ und $B^{\sigma}$ es sind. Entsprechendes gilt für alle später als "Tensoren“ einzuführenden Systeme (Regel von der Addition und Subtraktion der Tensoren).

Kovarianter Vierervektor. Vier Größen $A_{v}$ nennen wir die Komponenten eines kovarianten Vierervektors, wenn für jede beliebige Wahl des kontravarianten Vierervektors $B^{v}$

$$
\sum_{\nu} A_{\nu} B^{\nu}=\text { Invariante. }
$$

Aus dieser Definition folgt das Transformationsgesetz des kovarianten Vierervektors. Ersetzt man nämlich auf der rechten Seite der Gleichung

$$
\sum_{\sigma} A_{\sigma}^{\prime} B^{\sigma^{\prime}}=\sum_{v} A_{v} B^{v}
$$

$B^{v}$ durch den aus der Umkehrung der Gleichung (5a) folgenden Ausdruck

$$
\sum_{\sigma} \frac{\partial x_{v}}{\partial x_{\sigma}{ }^{\prime}} B^{\sigma^{\prime}}
$$

so erhält man

$$
\sum_{\sigma} B^{\sigma^{\prime}} \sum_{\nu} \frac{\partial x_{v}}{\partial x_{\sigma}^{\prime}} A_{\nu}=\sum_{\sigma} B^{\sigma^{\prime}} \cdot A_{\sigma}^{\prime} .
$$


Hieraus folgt aber, weil in dieser.Gleichung die $B^{o^{\prime}}$ unabhängig voneinander frei wählbar sind, das Transformationsgesetz

$$
A_{\sigma}^{\prime}=\sum \frac{\partial x_{v}}{\partial x_{\sigma}} A_{v} .
$$

Bemerkung zur Vereinfachung der Schreibweiseder Ausdrücke.

Ein Blick auf die Gleichungen dieses Paragraphen zeigt, daß über Indizes, die zweimal unter einem Summenzeichen auftreten $[\mathrm{z}$. B. der Index $v$ in (5)], stets summiert wird, und zwar nur über zweimal auftretende Indizes. Es ist deshalb möglich, ohne die Klarheit zu beeinträchtigen, die Summenzeichen wegzulassen. Dafür führen wir die Vorschrift ein: Tritt ein Index in einem Term eines Ausdruckes zweimal auf, so ist über ihn stets zu summieren, wenn nicht ausdrücklich das Gegenteil bemerkt ist.

Der Unterschied zwischen dem kovarianten und kontravarianten Vierervektor liegt in dem Transformationsgesetz [(7) bzw. (5)]. Beide Gebilde sind Tensoren im Sinne der obigen allgemeinen Bemerkung; hierin liegt ihre Bedeutung. Im Anschlu $\beta$ an Ricei und Levi-Civita wird der kontravariante Charakter durch oberen, der kovariante durch unteren Index bezeichnet.

\section{\& 6. Tensoren $\mathbf{z w}$ eiten und höheren Ranges.}

Kontravarianter Tensor. Bilden wir sämtliche 16 Produkte $A^{\mu \nu}$ der Komponenten $A^{\mu}$ und $B^{\nu}$ zweier kontravarianten Vierervektoren

(8)

$A^{\mu \nu}=A^{\mu} B^{\nu}$,

so erfüllt $A^{\mu \nu}$ gemäß (8) und (5a) das Transformationsgesetz

$$
A^{\sigma x^{\prime}}=\frac{\partial x_{\sigma}^{\prime}}{\partial x_{\mu}} \frac{\partial x_{r}^{\prime}}{\partial x_{\nu}} A^{\mu \nu} \text {. }
$$

Wir nennen ein Ding, das bezüglich eines jeden Bezugssystems durch 16 Größen (Funktionen) beschrieben wird, die das Transformationsgesetz (9) erfüllen, einen kontravarianten Tensor zweiten Ranges. Nicht jeder solcher Tensor läßt sich gemä $\beta$ (8) aus zwei Vierervektoren bilden. Aber es ist leicht zu beweisen, da $B$ sich 16 beliebig gegebene $A^{\mu \nu}$ darstellen lassen als die Summe der $A^{\mu} B^{\nu}$ von vier geeignet gewählten
Paaren von Vierervektoren. Deshalb kann man beinahe alle Sätze, die für den durch (9) definierten Tensor zweiten Ranges gelten, am einfachsten dadurch beweisen, daß man sie für spezielle Tensoren vom Typus (8) dartut.

Kontravarianter Tensor beliebigen Ranges. Es ist klar, daß man entsprechend (8) und (9) auch kontravariante Tensoren dritten und höheren Ranges definieren kann mit $\mathbf{4}^{\mathbf{3}}$ usw. Komponenten. Ebenso erhellt aus (8) und (9), daß man in diesem Sinne den kontravarianten Vierervektor als kontravarianten Tensor ersten Ranges auffassen karn.

Kovarianter Tensor. Bildet man andererseits die 16 Produkte $A_{\mu \nu}$ der Komponenten zweier kovarianter Vierervektoren $A_{\mu}$ und $B_{v}$

$$
A_{\mu \nu}=A_{\mu} B_{\nu},
$$

so gilt für diese das Transformationsgesetz

$$
A_{\sigma \tau}{ }^{\prime}=\frac{\partial x_{\mu}}{\partial x_{\sigma}{ }^{\prime}} \frac{\partial x_{v}}{\partial x_{\tau}{ }^{\prime}} A_{\mu \nu} .
$$

Durch dieses Transformationsgesetz wird der kovariante Tensor zweiten Ranges definiert. Alle Bemerkungen, welche vorher über die kontravarianten Tensoren gemacht wurden, gelten auch für die kovarianten Tensoren.

Bemerkung. Es ist bequem, den Skalar (Invariante) sowohl als kontravarianten wie als kovarianten Tensor vom Range Null zu behandeln.

Gemischter Tensor. Man kann auch einen Tensor zweiten Ranges vom Typus

$$
A_{\mu}{ }^{v}=A_{\mu} B^{v}
$$

definieren, der bezüglich des Index $\mu$ kovariant, bezüglich des Index $v$ kontravariant ist. Sein Transformationsgesetz ist

$$
A_{\sigma}{ }^{\prime}=\frac{\partial x_{\tau}^{\prime}}{\partial x_{\beta}} \frac{\partial x_{a}}{\partial x_{\sigma}^{\prime}}, A_{a}^{\beta} .
$$

Natürlich gibt es gemischte Tensoren mit beliebig vielen Indizes kovarianten und beliebig vielen Indizes kontravarianten Charakters. Der kovariante und der kontravariante Tensor können als spezielle Fälle des gemischten angesehen werden. 


$$
-22-
$$

Symmetrische Tensoren. Ein kontravarianter bzw. kovarianter Tensor zweiten oder höheren Ranges heißt sym metrisch, wenn zwei Komponenten, die durch Vertauschung irgend zweier Indizes auseinander hervorgehen, gleich sind. Der Tensor $A^{\mu \nu}$ bzw. $A_{\mu \nu}$ ist also symmetrisch, wenn für jede Kombination der Indizes

bzw.

ist.

$$
A^{\mu v}=A^{v \mu}
$$

$$
A_{\mu \nu}=A_{v u}
$$

Es $m u B$ bewiesen werden, da $B$ die so definierte Symmetrie eine vom Bezugssystem unabhängige Eigensehaft ist. (Aus (9) folgt in der Tat mit Rücksicht auf (14)

$$
A^{\sigma r^{\prime}}=\frac{\partial x_{\sigma}^{\prime}}{\partial x_{\mu}} \frac{\partial x_{q}^{\prime}}{\partial x_{v}} A^{\mu v}=\frac{\partial x_{a}^{\prime}}{\partial x_{\mu}} \frac{\partial x_{q}^{\prime}}{\partial x_{v}} A^{v \mu}=\frac{\partial x_{q}^{\prime}}{\partial x_{\mu}} \frac{\partial x_{a}^{\prime}}{\partial x_{v}} A^{\mu v}=A^{r o^{\prime}} .
$$

Die vorletzte Gleichsetzung beruht auf der Vertauschung der Summationsindizes $\mu$ und $\boldsymbol{\nu}$ (d. h. auf bloßer Änderung der Bezeichnungsweise).

Antisymmetrische Tensoren. Ein kontravarianter bzw. kovarianter Tensor zweiten, dritten oder vierten Ranges heißt antisymmetrisch, wenn zwei Komponenten, die durch Vertauschung irgend zweier Indizes auseinander hervorgehen, entgegengesetzt gleich sind. Der Tensor $A^{\mu \nu}$ bzw. $A_{\mu \nu}$ ist also antisymmetrisch, wenn stets

$$
A^{\mu \nu}=-A^{\nu \mu},
$$

bzw.

(15 a)

ist.

$$
A_{\mu \nu}=-A_{v \mu}
$$

Von den 16 Komponenten $A^{\mu \nu}$ verschwinden die vier Komponenten $A^{\mu \mu}$; die übrigen sind paarweise entgegengesetzt gleich, so daB nur 6 numerisch verschiedene Komponenten vorhanden sind (Sechservektor). Ebenso sieht man, daB der antisymmetrische Tensor $A^{\mu \nu \sigma}$ (dritten Ranges) nur vier numerisch verschiedene Komponenten hat, der antisymmetrische Tensor $A^{\mu \nu \sigma \tau}$ nur eine einzige. Symmetrische Tensoren höheren als vierten Ranges gibt es in einem Kontinuum von vier Dimensionen nicht.

\section{\& 7. Multiplikation der Tensoren.}

Äußere Multiplikation der Tensoren. Man erhält aus den Komponenten eines Tensors vom Range $z$ und eines solchen vom Range $z^{\prime}$ die Komponenten eines Tensors vom Range $z+z^{\prime}$, indem man alle Komponenten des ersten mit allen Komponenten des zweiten paarweise multipliziert. So entstehen beispielsweise die Tensoren $T$ aus den Tensoren $A$ und $B$ verschiedener Art

$$
\begin{aligned}
& T_{\mu \nu \sigma}=A_{\mu \nu} B_{\sigma}, \\
& T^{a \beta \gamma \delta}=A^{\alpha \beta} B^{\gamma \delta}, \\
& T_{\alpha \beta}^{\gamma \delta}=A_{a \beta} B^{\gamma \delta} .
\end{aligned}
$$

Der Beweis des Tensorcharakters der $T$ ergibt sich unmittelbar aus den Darstellungen (8), (10), (12) oder aus den Transformationsregeln (9), (11), (13). Die Gleichungen (8), (10), (12) sind selbst Beispiele äußerer Multiplikation (von Tensoren ersten Ranges).

"Verjüngung" eines gemischten Tensors. Aus jedem gemischten Tensor kann ein Tensor von einem um zwei kleineren Range gebildet werden, indem man einen Index kovarianten und einen Index kontravarianten Charakters gleichsetzt und nach diesem Index summiert (,,Verjüngung"). Man gewinnt so z. B. aus dem gemischten Tensor vierten Ranges $A_{a \beta}^{\gamma \delta}$ den gemischten Tensor zweiten Ranges

$$
A_{\beta}^{\delta}=A_{a \beta}^{a \delta}\left(=\sum_{\alpha} A_{\alpha \beta}^{a \delta}\right)
$$

und aus diesem, abermals durch Verjüngung, den Tensor nullten Ranges $A=A_{\beta}^{\beta}=A_{\alpha \beta}^{\alpha \beta}$.

Der Beweis dafür, da $\beta$ das Ergebnis der Verjüngung wirklich Tensorcharakter besitzt, ergibt sich entweder aus der Tensordarstellung gemäß der Verallgemeinerung von (12) in Verbindung mit (6) oder aus der Verallgemeinerung von (13).

Innere und gemischte Multiplikation der Tensoren. Diese bestehen in der Kombination der äußeren Multiplikation mit der Verjüngung.

Beispiele: - Aus dem kovarianten Tensor zweiten Ranges $A_{\mu \nu}$ und dem kontravarianten Tensor ersten Ranges $B^{\sigma}$ bilden wir durch äußere Multiplikation den gemischten Tensor

$$
D_{\mu \nu}^{\sigma}=A_{\mu \nu} B^{\sigma} \text {. }
$$


Durch Verjüngung nach den Indizes $\boldsymbol{\nu}, \boldsymbol{\sigma}$ entsteht der kovariante Vierervektor

$$
D_{\mu}=D_{\mu \nu}^{\nu}=A_{\mu \nu} B^{\nu} .
$$

Diesen bezeichnen wir auch als inneres Produkt der Tensoren $A_{\mu \nu}$ und $B^{\sigma}$. Analog bildet man aus den Tensoren $A_{\mu \nu}$ und $B^{\sigma \tau}$ durch äußere Multiplikation und zweimalige Verjüngung das innere Produkt $A_{\mu \nu} B^{\mu \nu}$. Durch äußere Produktbildung und einmalige Verjüngung erhält man aus $A_{\mu \nu}$ und $B^{o r}$ den gemischten Tensor zweiten Ranges $D_{\mu}^{\tau}=A_{\mu \nu} B^{\nu \tau}$. Man kann diese Operation passend als eine gemischte bezeichnen; denn sie ist eine äußere bezüglich der Indizes $\boldsymbol{\mu}$ und $\boldsymbol{\tau}$, eine innere bezüglich der Indizes $\boldsymbol{v}$ und $\boldsymbol{\sigma}$.

Wir beweisen nun einen Satz, der zum Nachweis des Tensorcharakters oft verwendbar ist. Nach dem soeben Dargelegten ist $A_{\mu \nu} B^{\mu \nu}$ ein Skalar, wenn $A_{\mu \nu}$ und $B^{\sigma \tau}$ Tensoren sind. Wir behaupten aber auch folgendes. Wenn $A_{\mu \nu} B^{\mu \nu}$ für jede Wahl des Tensors $B^{\mu v}$ eine Invariante ist, so hat $A_{\mu \nu}$ Tensorcharakter.

Beweis. - Es ist nach Voraussetzung für eine beliebige Substitution

$$
A_{\sigma \tau}{ }^{\prime} B^{\sigma r^{\prime}}=A_{\mu \nu} B^{\mu \nu} \text {. }
$$

Nach der Umkehrung von (9) ist aber

$$
B^{\mu \nu}=\frac{\partial x_{\mu}}{\partial x_{\sigma}^{\prime}} \frac{\partial x_{v}}{\partial x_{r}^{\prime}} B^{n r^{\prime}} .
$$

Dies, eingesetzt in obige Gleichung, liefert:

$$
\left(A_{\sigma \tau}^{\prime}-\frac{\partial x_{\mu}}{\partial x_{\sigma}^{\prime}} \frac{\partial x_{v}}{\partial x_{\tau}^{\prime}} A_{\mu \nu}\right) B^{o r^{\prime}}=0 \text {. }
$$

Dies kann bei beliebiger Wahl von $B^{o \tau^{\prime}}$ nur dann erfüllt sein, wenn die Klammer verschwindet, woraus mit Rücksicht auf (11) die Behauptung folgt.

Dieser Satz gilt entsprechend für Tensoren beliebigen Ranges und Charakters; der Beweis ist stets analog zu führen.

Der Satz läßt sich ebenso beweisen in der Form: Sind $B^{\mu}$ und $C^{\nu}$ beliebige Vektoren, und ist bei jeder Wahl derselben das innere Produkt

$$
A_{\mu \nu} B^{\mu} C^{\nu}
$$

ein Skalar, so ist $A_{\mu \nu}$ ein kovarianter Tensor. Dieser letztere Satz gilt auch dann noch, wenn nur die speziellere Aussage zutrifft, da $\beta$ bei beliebiger Wahl des Vierervektors $B^{\mu}$ das skalare Produkt

$$
A_{\mu \nu} B^{\mu} B^{\nu}
$$

ein Skalar ist, falls man außerdem weiß, daß $A_{\mu \nu}$ der Symmetriebedingung $A_{\mu \nu}=A_{\nu \mu}$ genügt. Denn auf dem vorhin angegebenen Wege beweist man den Tensorcharakter von $\left(A_{\mu \nu}+A_{\nu \mu}\right)$, woraus dann wegen der Symmetrieeigenschaft der Tensorcharakter von $A_{u v}$ selbst folgt. Auch dieser Satz läBt sich leicht verallgemeinern auf den Fall kovarianter und kontravarianter Tensoren beliebigen Ranges.

Endlich folgt aus dem Bewiesenen der ebenfalls auf beliebige Tensoren zu verallgemeinernde Satz: Wenn die Größen $A_{\mu \nu} B^{\nu}$ bei beliebiger Wahl des Vierervektors $B^{\nu}$ einen Tensor ersten Ranges bilden, so ist $A_{\mu \nu}$ ein Tensor zweiten Ranges. Ist nämlich $C^{\mu}$ ein beliebiger Vierervektor, so ist wegen des Tensorcharakters $A_{\mu \nu} B^{\nu}$ das innere Produkt $A_{\mu \nu} C^{\mu} B^{\nu}$ bei beliebiger Wahl der beiden Vierervektoren $C^{\mu}$ und $B^{\nu}$ ein Skalar, woraus die Behauptung folgt.

\section{\$ 8. Finiges über den Fundamentaltensor der $g_{\mu \nu}$.}

Der kovariante Fundamentaltensor. In dem invarianten Ausdruck des Quadrates des Linienelementes

$$
d s^{2}=g_{\mu \nu} d x_{\mu} d x_{\nu}
$$

spielt $d x_{\mu}$ die Rolle eines beliebig wählbaren kontravarianten Vektors. Da ferner $g_{\mu \nu}=g_{v \mu}$, so folgt nach den Betrachtungen des letzten Paragraphen hieraus, daß $g_{\mu \nu}$ ein kovarianter Tensor zweiten Ranges ist. Wir nennen ihn "Fundamentaltensor". Im folgenden leiten wir einige Eigenschaften dieses Tensors ab, die zwar jedem Tensor zweiten Ranges eigen sind; aber die besondere Rolle des Fundamentaltensors in unserer Theorie, welche in der Besonderheit der Gravitationswirkungen ihren physikalischen Grund hat, bringt es mit sich, daß die zu entwickelnden Relationen nur bei dem Fundamentaltensor für uns von Bedeutung sind.

Der kontravariante Fundamentaltensor. Bildet man in dem Determinantenschema der $g_{\mu \nu}$ zu jedem $g_{\mu \nu}$ die Unterdeterminante und dividiert diese durch die Determinante $g=\left|g_{\mu \nu}\right|$ der $g_{\mu \nu}$, so erhält man gewisse Größen $g^{\mu \nu}\left(=g^{\nu \mu}\right)$, von denen wir beweisen wollen, daß sie einen kontravarianten T'ensor bilden. 


$$
-26-
$$

Nach einem bekannten Determinantensatze ist

$$
g_{\mu \sigma} g^{\nu \sigma}=\delta_{\mu}{ }^{\nu},
$$

wobei das Zeichen $\delta_{\mu}{ }^{\nu} 1$ oder 0 bedeutet, je nachdem $\mu=\nu$ oder $\mu \neq \nu$ ist. Statt des obigen Ausdruckes für $d s^{2}$ können wir auch

oder nach (16) auch

$$
g_{\mu \sigma} \delta_{\nu}{ }^{\sigma} d x_{\mu} d x_{\nu}
$$

$$
g_{\mu \sigma} g_{v \tau} g^{\sigma \tau} d x_{\mu} d x_{\nu}
$$

schreiben. Nun bilden aber nach den Multiplikationsregeln des vorigen Paragraphen die Größen

$$
d \xi_{\sigma}=g_{\mu \sigma} d x_{\mu}
$$

einen kovarianten Vierervektor, und zwar (wegen der willkürlichen Wählbarkeit der $d x_{\mu}$ ) einen beliebig wählbaren Vierervektor. Indem wir ihn in unseren Ausdruck einführen, erhalten wir

$$
d s^{2}=g^{\sigma \tau} d \xi_{\sigma} d \xi_{\tau} .
$$

Da dies bei beliebiger Wahl des Vektors $d \xi_{\sigma}$ ein Skalar ist und $g^{\sigma \tau}$ nach seiner Definition in den Indizes $\sigma$ und $\tau$ symmetrisch ist, folgt aus den Ergebnissen des vorigen Paragraphen, da $\beta g^{\sigma \tau}$ ein kontravarianter Tensor ist. Aus (16) folgt noch, daß auch $\delta_{\mu}{ }^{v}$ ein Tensor ist, den wir den gemischten Fundamentaltensor nennen können.

Determinante des Fundamentaltensors. Nach dem Multiplikationssatz der Determinanten ist

Andererseits ist

$$
\left|g_{\mu a} g^{a v}\right|=\left|g_{\mu a}\right|\left|g^{a v}\right| \text {. }
$$

Also folgt

$$
\left|g_{u a} g^{\alpha \nu}\right|=\left|\delta_{\mu} v\right|=1 .
$$

$$
\left|g_{\mu \nu}\right|\left|g^{\mu \nu}\right|=1 \text {. }
$$

Invariante des Volumens. Wir suchen zuerst das Transformationsgesetz der Determinante $g=\left|g_{\mu \nu}\right|$. GemäB (11) ist

$$
g^{\prime}=\left|\frac{\partial x_{\mu}}{\partial x_{\sigma}^{\prime}} \frac{\partial x_{\nu}}{\partial x_{\tau}^{\prime}} g_{\mu \nu}\right| \text {. }
$$

Hieraus folgt durch zweimalige Anwendung des Multiplikationssatzes der Determinanten

$$
g^{\prime}=\left|\frac{\partial x_{\mu}}{\partial x_{\sigma}^{\prime}}\right|\left|\frac{\partial x_{v}}{\partial x_{\tau}^{\prime}}\right|\left|g_{u \nu}\right|=\left|\frac{\partial x_{\mu}}{\partial x_{\sigma}^{\prime}}\right|^{2} g,
$$

oder

$$
\sqrt{g^{\prime}}=\left|\frac{\partial x_{\mu}}{\partial x_{\sigma}^{\prime}}\right| \sqrt{g}
$$

Andererseits ist das Gesetz der Transformation des Volumelementes

$$
d \tau^{\prime}=\int d x_{1} d x_{2} d x_{3} d x_{4}
$$

nach dem bekannten Jako bischen Satze

$$
d \boldsymbol{\tau}^{\prime}=\left|\frac{\partial x_{\sigma}^{\prime}}{\partial x_{\mu}}\right| d \tau
$$

Durch Multiplikation der beiden letzten Gleichungen erhält man

$$
\sqrt{g^{\prime}} d \tau^{\prime}=\sqrt{g} d \tau
$$

Statt $\sqrt{g}$ wird im folgenden die Größe $\sqrt{-g}$ eingeführt, welche wegen des hyperbolischen Charakters des zeiträumlichen Kontinuums stets einen reellen Wert hat. Die Invariante $\sqrt{-g} d \tau$ ist gleich der Größe des im ,örtlichen Bezugssystem“ mit starren Maßstäben und Uhren im Sinne der speziellen Relativitätstheorie gemessenen vierdimensionalen Volumelementes.

Bemerkung über den Charakter des raumzeitlichen Kontinuums. Unsere Voraussetzung, $\mathrm{da} B$ im unendlich Kleinen stets die spezielle Relativitätstheorie gelte, bringt es mit sich, daß sich $d s^{2}$ immer gemäß (1) durch die reellen Größen $d X_{1} \ldots d X_{4}$ ausdrücken läßt. Nennen wir $d \tau_{0}$ das ,natürliche" Volumelement $d X_{1} d X_{2} d X_{3} d X_{4}$, so ist also

$$
d \tau_{0}=\sqrt{-g} d \tau \text {. }
$$

Soll an einer Stelle des vierdimensionalen Kontinuums $\sqrt{-g}$ verschwinden, so bedeutet dies, da $\beta$ hier einem endlichen Koordinatenvolumen ein unendlich kleines „natürliches“ Volumen entspreche. Dies möge nirgends der Fall sein. Dann kann $g$ sein Vorzeichen nicht ändern; wir werden im Sinne der speziellen Relativitätstheorie annehmen, daß $g$ stets einen endlichen negativen Wert habe. Es ist dies eine Hypothese über die physikalische Natur des betrachteten Kontinuums und gleichzeitig eine Festsetzung über die Koordinatenwahl. 
Ist aber $-g$ stets positiv und endlich, so liegt es nahe, die Koordinatenwahl a posteriori so zu treffen, daß diese Größe gleich 1 wird. Wir werden später sehen, daß durch eine solche Beschränkung der Koordinatenwahl eine bedeutende Vereinfachung der Naturgesetze erzielt werden kann. An Stelle von (18) tritt dann einfach

$$
d \tau^{\prime}=d \tau,
$$

woraus mit Rücksicht auf Jakobis Satz folgt

$$
\left|\frac{\partial x_{\sigma}^{\prime}}{\partial x_{\mu}}\right|=1 \text {. }
$$

Bei dieser Koordinatenwahl sind also nur Substitutionen der Koordinaten von der Determinante 1 zulässig.

Es wäre aber irrtümlich, zu glauben, daß dieser Schritt einen partiellen Verzicht auf das allgemeine Relativitätspostulat bedeute. Wir fragen nicht: „Wie heißen die Naturgesetze, welche gegenüber allen Transformationen von der Determinante 1 kovariant sind ?" Sondern wir fragen: „Wie heißen die allgemein kovarianten Naturgesetze?" Erst nachdem wir diese aufgestellt haben, vereinfachen wir ihren Ausdruck durch eine besondere Wahl des Bezugssystems.

Bildung neuer Tensoren vermittelst des Fundamentaltensors. Durch innere, äußere und gemischte Multiplikation eines Tensors mit dem Fundamentaltensor entstehen Tensoren anderen Charakters und Ranges.

Beispiele:

$$
\begin{aligned}
& A^{\mu}=g^{\mu \sigma} A_{\sigma}, \\
& A=g_{\mu \nu} A^{\mu v} .
\end{aligned}
$$

Besonders sei auf folgende Bildungen hingewiesen:

$$
\begin{aligned}
& A^{\mu \nu}=g^{\mu \alpha} g^{\nu \beta} A_{\alpha \beta}, \\
& A_{\mu \nu}=g_{\mu \alpha} g_{\nu \beta} A^{\alpha \beta}
\end{aligned}
$$

(,Ergänzung“ des kovarianten bzw. kontravarianten Tensors) und

$$
B_{\mu v}=g_{\mu v} g^{\alpha \beta} A_{\alpha \beta} .
$$

Wir nennen $B_{\mu \nu}$ den zu $A_{u \nu}$ gehörigen reduzierten Tensor. Analog

$$
B^{\mu \nu}=g^{\mu \nu} g_{a \beta} A^{\alpha \beta}
$$

Es sei bemerkt, daß $g^{\mu v}$ nichts anderes ist als die Ergänzung von $g_{\mu \nu}$. Denn man hat

$$
g^{\mu a} g^{\nu \beta} g_{a \beta}=g^{\mu a} \delta_{a}^{\nu}=g^{\mu \nu} .
$$

\section{\$ 9. Gleichung der geodätischen Linie (bzw. der Punkt-} bewegung).

Da das „Linienelement“ $d s$ eine unabhängig vom Koordinatensystem definierte Größe ist, hat auch die zwischen zwei Punkten $P_{1}$ und $P_{2}$ des vierdimensionalen Kontinuums gezogene Linie, für welche $\int d s$ ein Extremum ist (geodätische Linie), eine von der Koordinatenwahl unabhängige Bedeutung. Ihre Gleichung ist

$$
\delta\left\{\int_{P_{1}}^{P_{2}} d s\right\}=0 .
$$

Aus dieser Gleichung findet man in bekannter Weise durch Ausführung der Variation vier totale Differentialgleichungen, welche diese geodätische Linie bestimmen; diese Ableitung soll der Vollständigkeit halber hier Platz finden. Es sei $\lambda$ eine Funktion der Koordinaten $x_{v}$; diese definiert eine Schar von Flächen, welche die gesuchte geodätische Linie sowie alle ihr unendlich benachbarten, durch die Punkte $P_{1}$ und $P_{\mathbf{2}}$ gezogenen Linien schneiden. Jede solche Kurve kann dann dadurch gegeben gedacht werden, daß ihre Koordinaten $x_{v}$ in Funktion von $\lambda$ ausgedrückt werden. Das Zeichen $\delta^{\nu}$ entspreche dem Übergang von einem Punkte der gesuchten geodätischen Linie zu demjenigen Punkte einer benachbarten Kurve, welcher zu dem nämlichen $\lambda$ gehört. Dann läßt sich (20) durch

$$
\left\{\begin{array}{l}
\int_{\lambda_{1}}^{\lambda_{2}} \delta w d \lambda=0 \\
w^{2}=g_{\mu \nu} \frac{d x_{\mu}}{d \lambda} \frac{d x_{\nu}}{d \lambda}
\end{array}\right.
$$

ersetzen. Da aber

$$
\delta \cdot w=\frac{1}{w}\left\{\frac{1}{2} \frac{\partial g_{\mu \nu}}{\partial x_{\sigma}} \frac{d x_{\mu}}{d \lambda} \frac{d x_{\nu}}{d \lambda} \delta x_{\sigma}+g_{\mu \nu} \frac{d x_{\mu}}{d \lambda} \delta\left(\frac{d x_{\nu}}{d \lambda}\right)\right\},
$$

so erhält man nach Einsetzen von $\delta w$ in (20a) mit Rücksicht darauf, daß

$$
\delta\left(\frac{d x_{v}}{d \lambda}\right)=\frac{d \delta x_{v}}{d \lambda}
$$


nach partieller Integration

$$
\left\{\begin{array}{l}
\int_{\lambda_{1}}^{\lambda_{2}} d \lambda x_{\sigma} \delta x_{\sigma}=0 \\
x_{\sigma}=\frac{d}{d \lambda}\left\{\frac{g_{\mu \nu}}{w} \frac{d x_{\mu}}{\partial \lambda}\right\}-\frac{1}{2 w} \frac{\partial g_{\mu \nu}}{\partial x_{\sigma}} \frac{d x_{\mu}}{d \lambda} \frac{d x_{\nu}}{d \lambda} .
\end{array}\right.
$$

Hieraus folgt wegen der freien Wählbarkeit der $\delta x_{\sigma}$ das Verschwinden der $\boldsymbol{x}_{\sigma}$. Also sind

$$
\boldsymbol{x}_{\sigma}=0
$$

die Gleichungen der geodätischen Linie. Ist auf der betrachteten geodätischen Linie nicht $d s=0$, so können wir als Parameter $\lambda$ die auf der geodätischen Linie gemessene „Bogenlänge" $s$ wählen. Dann wird $w=1$, und man erhält an Stelle von $(20 \mathrm{c})$

$$
g_{\mu \nu} \frac{d^{2} x_{\mu}}{d s^{2}}+\frac{\partial g_{\mu \nu}}{\partial x_{\sigma}} \frac{d x_{\sigma}}{d s} \frac{d x_{\mu}}{d s}-\frac{1}{2} \frac{\partial g_{\mu \nu}}{\partial x_{\sigma}} \frac{d x_{\mu}}{d s} \frac{d x_{v}}{d s}=0,
$$

oder durch bloße Änderung der Bezeichnungsweise

$$
g_{a \sigma} \frac{d^{2} x_{u}}{d s^{2}}+\left[\begin{array}{c}
\mu \nu \\
\sigma
\end{array}\right] \frac{d x_{\mu}}{d s} \frac{d x_{\nu}}{d s}=0,
$$

wobei nach Christoffel gesetzt ist

$$
\left[\begin{array}{c}
\mu \nu \\
\sigma
\end{array}\right]=\frac{1}{2}\left(\frac{\partial g_{\mu \sigma}}{\partial x_{\nu}}+\frac{\partial q_{\nu \sigma}}{\partial x_{\mu}}-\frac{\partial g_{\mu \nu}}{\partial x_{\sigma}}\right) \text {. }
$$

Multipliziert man endlich $(20 \mathrm{~d})$ mit $g^{\sigma \tau}$ (äußere Multiplikation bezüglich $\tau$, innere bezüglich $\sigma$ ), so erhält man schließlich als endgültige Form der Gleichung der geodätischen Linie

$$
\frac{d^{2} x_{\tau}}{d s^{2}}+\left\{\begin{array}{c}
\mu \nu \\
\tau
\end{array}\right\} \frac{d x_{\mu}}{d s} \frac{d x_{v}}{d s}=0
$$

Hierbei ist nach Christoffel gesetzt

$$
\left\{\begin{array}{c}
\mu \nu \\
\tau
\end{array}\right\}=g^{\tau \alpha}\left[\begin{array}{c}
\mu \nu \\
\alpha
\end{array}\right] \text {. }
$$

\section{\& 10. Die Bildung von Tensoren durch Differentiation.}

Gestützt auf die Gleichung der geodätischen Linie können wir nun leicht die Gesetze ableiten, nach welchen durch Differentiation aus Tensoren neue Tensoren gebildet werden können. Dadurch werden wir erst in den Stand gesetzt, allgemein kovariante Differentialgleichungen aufzustellen. Wir erreichen dies Ziel durch wiederholte Anwendung des folgenden einfachen Satzes.

Ist in unserem Kontinuum eine Kurve gegeben, deren Punkte durch die Bogendistanz $s$ von einem Fixpunkt auf der Kurve charakterisiert sind, ist ferner $\varphi$ eine invariante Raumfunktion, so ist auch $d \varphi / d s$ eine Inváriante. Der $\mathrm{Be}$ weis liegt darin, daß sowohl $d \varphi$ als auch $d s$ Invariante sind.

$\mathrm{Da}$

$$
\frac{d \varphi}{d s}=\frac{\partial \varphi}{\partial x_{\mu}} \frac{d x_{\mu}}{d s}
$$

so ist auch

$$
\psi=\frac{\partial \varphi}{\partial x_{\mu}} \frac{d x_{\mu}}{d s}
$$

eine Invariante, und zwar für alle Kurven, die von einem Punkte des Kontinuums ausgehen, d. h. für beliebige Wahl des Vektors der $d x_{u}$. Daraus folgt unmittelbar, daß

$$
A_{\mu}=\frac{\partial p}{\partial x_{\mu}}
$$

ein kovarianter Vierervektor ist (Gradient von $\varphi$ ).

Nach unserem Satze ist ebenso der auf einer Kurve ge. nommene Differentialquotient

$$
\chi=\frac{d \psi}{d s}
$$

eine Invariante. Durch Einsetzen von $\psi$ erhalten wrr zunächst

$$
\chi=\frac{\partial^{2} \varphi}{\partial x_{\mu} \partial x_{\nu}} \frac{d x_{\mu}}{d s} \frac{d x_{v}}{d s}+\frac{\partial \varphi}{\partial x_{\mu}} \frac{d^{2} x_{\mu}}{d s^{2}} .
$$

Hieraus läßt sich zunächst die Existenz eines Tensors nicht ableiten. Setzen wir nun aber fest, daß die Kurve, auf welcher wir differenziiert haben, eine geodätische Kurve sei, so erhalten wir nach (22) durch Ersetzen von $d^{2} x_{v} / d s^{2}$.

$$
\chi=\left\{\frac{\partial^{2} \varphi}{\partial x_{\mu} \dot{\partial} x_{\nu}}-\left\{\begin{array}{c}
\mu \nu \\
\tau
\end{array}\right\} \frac{\partial \varphi}{\partial x_{\tau}}\right\} \frac{d x_{\mu}}{d s} \frac{d x_{v}}{d s} .
$$

Aus der Vertauschbarkeit der Differentiationen nach $\mu$ und $v$ und daraus, daß gemäß (23) und (21) die Klammer $\left\{\begin{array}{c}\mu \nu \\ \tau\end{array}\right\}$ bezüglich $\mu$ und $v$ symmetrisch ist, folgt, daß der Klammerausdruck in $\mu$ und $v$ symmetrisch ist. Da man von einem Punkt des Kontinuums aus in beliebiger Richtung eine geodätische Linie ziehen kann, $d x_{\mu} / d s$ also ein Vierervektor mit 


$$
-32-
$$

frei wählbarem Verhältnis der Komponenten ist, folgt nach den Ergebnissen des $\$ 7$, daß

$$
A_{\mu \nu}=\frac{\dot{b}^{2} \varphi}{\partial x_{\mu} \partial x_{\nu}}-\left\{\begin{array}{c}
\mu, \nu \\
\tau
\end{array}\right\} \frac{\partial \varphi}{\partial x_{\tau}}
$$

ein kovarianter Tensor zweiten Ranges ist. Wir haben also das Ergebnis gewonnen: Aus dem kovarianten Tensor ersten Ranges

$$
A_{\mu}=\frac{\partial q}{\partial x_{u}}
$$

können wir durch Differentiation einen kovarianten Tensor zweiten Ranges

$$
A_{\mu \nu}=\frac{\partial A_{\mu}}{\partial x_{\nu}}-\left\{\begin{array}{c}
\mu \nu \\
\tau
\end{array}\right\} d_{\tau}
$$

bilden. Wir nennen den Tensor $A_{\mu \nu}$ die „Erweiterung“ des Tensers $A$ Zunächst können wir leicht zeigen, daß diese Tensors $A_{\mu}$. Zunächst kônnen Tensor führt, wenn der Vektor $A_{\mu}$ Bildung auch dann auf einen Tensor führt, wenn der Vektor $A_{\mu}$
nicht als ein Gradient darstellbar ist. Um dies einzusehen, bemerken wir zunächst, daß

$$
\psi \frac{\partial \varphi}{\partial x_{\mu}}
$$

in Vierervektor ist, wenn $\psi$ und $\varphi$ Skalare sind. ein kovarianter Vierervektor ist, wenn $\varphi$ undern Dies ist auch der Fall für eine aus vier solchen Gliedern bestehende Summe

$S_{\mu}=\psi^{(1)} \frac{\partial q^{(1)}}{\partial x_{\mu}}+\cdot+\cdot+\psi^{(4)} \frac{\partial \varphi^{(4)}}{\partial x_{\mu}}$,

falls $\psi^{(1)} \varphi^{(1)} \ldots \ldots \psi^{(4)} \psi^{(4)}$ Skalare sind. Nun ist aber klar, daß Vierervektor in der Form $S_{\mu}$ darstellen sich jeder kovariante Vierervektor in der läBt. Ist nămlich $A_{\mu}$ ein Vierervektor, dessen beliebig gegebene Funktionen der $x_{v}$ sind, so hat metzen (bezüglich des gewählten Koordinatensystems) zu setzen

(bezüglich des gewählten $\quad \psi^{(1)}=x_{1}$,

$$
\begin{array}{ll}
\psi^{(1)}=A_{1}, & \psi^{(1)}=x_{1}, \\
\psi^{(2)}=A_{2}, & \varphi^{(2)}=x_{2}, \\
\psi^{(3)}=A_{3}, & \psi^{(3)}=x_{3}, \\
\psi^{(4)}=A_{4}, & \psi^{(4)}=x_{4},
\end{array}
$$

um zu erreichen, daB $S_{\mu}$ gleich $A_{\mu}$ wird.

da $B$, ein Tensor ist, wer̀n auf

Um daher zu beweisen, da $A_{\mu \nu}$ ein kovarianter Viererder rechten Seite für $A_{\mu}$ ein beliebiger ko zeigen, da $B$ dies vektor eingesetzt wird, brauchen wir nur zu zeigen, das dies
$-33-$

für den Vierervektor $S_{\mu}$ zutrifft. Für letzteres ist es aber, wie ein Blick auf die rechte Seite von (26) lehrt, hinreichend, den Nachweis für den Fall

$$
A_{\mu}=\psi \frac{\partial \varphi}{\partial x_{\mu}}
$$

zu führen. Es hat nun die mit $\psi$ multiplizierte rechte Seite von (25)

$$
\psi \frac{\partial^{2} \varphi}{\partial x_{\mu} \partial x_{\nu}}-\left\{\begin{array}{c}
\mu \nu \\
\tau
\end{array}\right\} \psi \frac{\partial \varphi}{\partial x_{\tau}}
$$

Tensorcharakter. Ebenso ist

$$
\frac{\partial \psi}{\partial x_{\mu}} \frac{\partial \varphi}{\partial x_{\nu}}
$$

ein Tensor (äußeres Produkt zweier Vierervektoren). Durch Addition folgt der Tensorcharakter von

$$
\frac{\partial}{\partial x_{\nu}}\left(\psi \frac{\partial \varphi}{\partial x_{\mu}}\right)-\left\{\begin{array}{c}
\mu \nu \\
\tau
\end{array}\right\}\left(\psi \frac{\partial \varphi}{\partial x_{\tau}}\right) \text {. }
$$

Damit ist, wie ein Blick auf (26) lehrt, der verlangte Nachweis für den Vierervektor

$$
\psi \frac{\partial \varphi}{\partial x_{\mu}}
$$

und daher nach dem vorhin Bewiesenen für jeden beliebigen Vierervektor $A_{\mu}$ geführt. -

Mit Hilfe der Erweiterung des Vierervektors kann man leicht die „Erweiterung“ eines kovarianten Tensors beliebigen Ranges definieren; diese Bildung ist eine Verallgemeinerung der Erweiterung des Vierervektors. Wir beschränken uns auf die Aufstellung der Erweiterung des Tensors zweiten Ranges, da dieser das Bildungsgesetz bereits klar übersehen läßt.

Wie bereits bemerkt, läßt sich jeder kovariante Tensor zweiten Ranges darstellen ${ }^{1}$ ) als eine Summe von Tensoren

1) Durch äußere Multiplikation der Vektoren mit den (beliebig gegebenen) Komponenten $A_{11}, A_{12}, A_{13}, A_{14}$ bzw. 1, 0, 0 , 0 entsteht ein Tensor mit den Komponenten

$$
\begin{array}{cccc}
A_{11} & A_{12} & A_{13} & A_{14} \\
0 & 0 & 0 & 0 \\
0 & 0 & 0 & 0 \\
0 & 0 & 0 & 0
\end{array}
$$

Durch Addition von vier Tensoren von diesem Typus erhält man den Tensor $A_{\mu \nu}$ mit beliebig vorgeschriebenen Komponenten,

Einstein. 
rom Typus $A_{n} B_{y}$. Es wird deshalb genügen, den Ausdruck der Erweiterung für einen solchen speziellen Tensor abzuleiten. Nach (26) haben die Ausdrücke

$$
\begin{aligned}
& \frac{\partial A_{\mu}}{\partial x_{\sigma}}-\left\{\begin{array}{c}
\sigma \mu \\
\tau
\end{array}\right\} A_{\tau}, \\
& \frac{\partial B_{v}}{\partial x_{\sigma}}-\left\{\begin{array}{c}
\sigma \nu \\
\tau
\end{array}\right\} B_{\tau}
\end{aligned}
$$

T'ensorcharakter. Durch äußere Multiplikation des ersten mit $B_{v}$, des zweiten mir $A_{\mu}$ erhält man je einen Tensor dritten Ranges; deren Addition ergibt den Tensor dritten Ranges

$$
A_{\mu v \sigma}=\frac{\partial A_{\mu v}}{\partial x_{\sigma}}-\left\{\begin{array}{c}
\sigma \mu \\
\tau
\end{array}\right\} A_{\tau \nu}-\left\{\begin{array}{c}
\sigma \nu \\
\tau
\end{array}\right\} A_{\mu \tau},
$$

wobei $A_{\mu \nu}=A_{u} B_{v}$ gesetzt ist. Da die rechte Seite von (27) linear und homogen ist bezüglich der $A_{\mu \nu}$ und deren ersten Ableitungen, führt dieses Bildungsgesetz nicht nur bei einem Tensor vom Typus $A_{u} B_{v}$, sondern auch bei einer Summe solcher Tensoren, d. h. bei einem beliebigen kovarianten 'Tensor zweiten Ranges, zu einem Tensor. Wir nennen $A_{\mu v a}$ die Erweiterung des Tensors $A_{\mu v}$.

Es ist klar, laß (26) und (24) nur spezielle Fälle von Erweiterung betreffen (Erweiterung des Tensors ersten bzw. nullten Ranges). Überhaupt lassen sich alle speziellen Bildungsgesetze von Tensoren auf (27) in Verbindung mit Tensormultiplikationen auffassen.

\section{\$11. Einige Spezialfälle von besonderer Bedeutung.}

Einige den Fiundamentaltensor betreffende Hilfssätze. Wir leiten zunächst einige im folgenden viel gebrauchte Hilfsgleichungen ab. Nach der Regel von der Differentiation der Determinanten ist

$$
d g=g^{\mu \nu} g d g_{\mu \nu}=-g_{\mu \nu} g d g^{\mu \nu} .
$$

l)ie letzte Form rechtfertigt sich durch die vorletzte, wenn man bedenkt, daß $g_{\mu \nu} g^{\mu^{\prime} v}=\delta_{\mu}^{\mu^{\prime}}$, daß also $g_{\mu v} g^{\mu v}=4$, folglich Aus $(28$, folgt

$$
g_{\mu \nu} \prime g^{\mu \nu}+g^{\mu \nu} d g_{\mu \nu}=0 \text {. }
$$

(29) $\frac{1}{V-y} \frac{\partial V-g}{\partial x_{a}}=\frac{1}{2} \frac{\partial \lg (-g)}{\partial x_{n}}=\frac{1}{2} g^{\mu \nu} \frac{\partial y_{\mu \nu}}{\partial x_{o}}=-\frac{1}{2} g_{\mu \nu} \frac{\partial g^{\mu \nu}}{\partial x_{o}}$.
Aus

$$
y_{\mu \sigma} g^{v \sigma}=\delta_{\mu}{ }^{v}
$$

folgt ferner durch Differentiation

$$
\text { (30) } \begin{cases}\text { bzw. } & g_{\mu \sigma} d g^{v \sigma}=-g^{\nu \sigma} d g_{\mu \sigma} \\ g_{\mu \sigma} \frac{\partial g^{\nu \sigma}}{\partial x_{\lambda}} & =-y^{\nu \sigma} \frac{\partial g_{\mu \sigma}}{\partial x_{\lambda}} .\end{cases}
$$

Durch gemischte Multiplikation mit $g^{\sigma \tau}$ bzw. $g_{v \text {. }}$ erhält man hieraus (bei geänderter Bezeichnungsweise der Indizes)

(31)

$$
\begin{aligned}
& d g^{\mu \nu}=-g^{\mu a} g^{\nu \beta} d g_{a \beta}, \\
& \frac{\partial g^{\mu \nu}}{\partial x_{\sigma}}=-g^{\mu a} g^{\nu \beta} \frac{\partial g_{a \beta}}{\partial x_{\sigma}}
\end{aligned}
$$

bzw.

(32)

$$
\begin{gathered}
d g_{\mu v}=-g_{\mu a} g_{\nu \beta} d g^{\alpha \beta} \\
\frac{\partial q_{\mu \nu}}{\partial x_{\sigma}}=-g_{\mu \alpha} g_{v \beta} \frac{\partial g^{a \beta}}{\partial x_{\sigma}}
\end{gathered}
$$

Die Beziehung (31) erlaubt eine Umformung, von der wir ebenfalls öfter Gebrauch zu machen haben. Gemäß (21) ist.

$$
\frac{\partial v_{a \beta}}{\partial x_{\sigma}}=\left[\begin{array}{cc}
\alpha & \sigma \\
\beta
\end{array}\right]+\left[\begin{array}{cc}
\beta & \sigma \\
\alpha
\end{array}\right] \text {. }
$$

Setzt man dies in die zweite der Formeln (31) ein, so erhält man mit Rücksicht auf (23)

$$
\frac{\partial g^{\mu r}}{\partial x_{\sigma}}=-\left(g^{\mu \tau}\left\{\begin{array}{c}
\tau \\
\nu
\end{array}\right\}+g^{\nu \tau}\left\{\begin{array}{cc}
\tau & \sigma \\
\mu
\end{array}\right\}\right)
$$

Durch Substitution der rechten Seite von (34) in (29) ergibt sich

$$
\frac{1}{\sqrt{-g}}=\frac{\partial \sqrt{-g}}{\partial x_{\sigma}}=\left\{\begin{array}{cc}
\mu & \sigma \\
\prime
\end{array}\right\}
$$

Divergenz des kontrdvarianten Vierervektors. Multipliziert man (26) mit dem kontravarianten Fundamentaltensor $g^{\mu v}$ (innere Multiplikation), so nimmt die rechte Seite nach Umformung des ersten Gliedes zunächst die Form an

$\frac{\partial}{\partial x_{v}}\left(g^{\mu \nu} A_{\mu}\right)-A_{\mu} \frac{\partial g^{\mu \nu}}{\partial x_{v}}-\frac{1}{2} g^{r a}\left(\frac{\partial g_{\mu \alpha}}{\partial x_{v}}+\frac{\partial g_{v a}}{\partial x_{\mu}}-\frac{\partial g_{\mu \nu}}{\partial x_{\alpha}}\right) g^{\mu v} A_{x}$.

Das letzte Glied dieses Ausdruckes kann gemäß (31) und (29) in die Form

$$
\frac{1}{2} \frac{\partial g^{\tau \nu}}{\partial x_{\nu}} A_{\tau}+\frac{1}{2} \frac{\partial g^{\tau \mu}}{\partial x_{\mu}} A_{\tau}+\frac{1}{\sqrt{-g}} \frac{\partial \sqrt{-g}}{\partial x_{a}} g^{\prime \prime v} A_{\tau} .
$$




$$
-36-
$$

rebracht werden. Da es auf die Benennung der Summations. hen sich die beiden ersten Glieder indizes nicht abigen weg; das letze lus obigen Ausdruckes vereinigen. Setzt man noch

$$
g^{\mu v} A_{\mu}=A^{v}
$$

wobei $A^{v}$ ebenso wie $A_{\mu}$ ein frei wählbarer Vektor ist, so erhält man endlich

$$
\Phi=\frac{1}{\sqrt{-g}} \frac{\partial}{\partial x_{\nu}}\left(\sqrt{-g} A^{v}\right) .
$$

Dieser Skalar ist die Divergenz des kontravarianten Vierervektors $A^{v}$.

Rotation" des (kovarianten) Vierervektors. Das zweite Glied in (26) ist in den Indizes $\mu$ und $v$ symmetrisch. Es ist (antisymmetrischer) Tensor. Man erhält

$$
B_{\mu \nu}=\frac{\partial A_{\mu}}{\partial x_{\nu}}-\frac{\partial A_{\nu}}{\partial x_{\mu}}
$$

Antisymmetrische Erweiterung eines Sechservektors. Wendet (27) anf einen antisymmetrischen Tensor zweiten Ranges (27) auf $A_{\mu \nu}$ an, bildet hierzu die beiden durchen der Indizes $\mu, v, \sigma$ entstehenden Gleichungen Ranges

(37) $B_{\mu \nu \sigma}=A_{\mu \nu \sigma}+A_{\nu \sigma \mu}+A_{\sigma \mu \nu}=\frac{\partial A_{\mu \nu}}{\partial x_{\sigma}}+\frac{\partial A_{\nu \sigma}}{\partial x_{\mu}}+\frac{\partial A_{\sigma \mu}}{\partial x_{\nu}^{\prime}}$, von welchem leicht $\mathrm{zu}$ beweisen ist, $\mathrm{da} B$ er antisymmetrisch ist. Divergenz des Sechservektors. Multipliziert man (27) mit $g^{\mu a} g^{\nu \beta}$ (gemischte Multiplikation), so erhält man ebenfalls einen Tensor. Das erste Glied der rechten Seite von (27) kann man in der Form

$$
\frac{\partial}{\partial x_{\sigma}}\left(g^{\mu a} g^{\nu \beta} A_{\mu \nu}\right)-g^{\mu a} \frac{\partial g^{\nu \beta}}{\partial x_{\sigma}} A_{\mu \nu}-g^{\nu \beta} \frac{\partial g^{\mu a}}{\partial x_{\sigma}} A_{\mu \nu}
$$

schreiben. Ersetzt man $g^{\mu a} g^{\nu \beta} A_{\mu \nu o}$ durch $A_{\sigma}^{a \beta}, g^{\mu a} g^{\nu \beta} A_{\mu \nu}$ durch $A^{\alpha \beta}$ und ersetzt man in dem umgeformten ersten Gliede

$$
\frac{\partial g^{\nu \beta}}{\partial x_{\sigma}} \text { und } \frac{\partial g^{\mu a}}{\partial x_{\sigma}}
$$

vermittelst (34), so entsteht aus der rechten Seite von (27) ein siebengliedriger Ausdruck, von dem sich vier Glieder wegheben. Es bleibt übrig

$$
A_{\sigma}^{\alpha \beta}=\frac{\partial A^{\alpha \beta}}{\partial x_{\sigma}}+\left\{\begin{array}{c}
\sigma x \\
\alpha
\end{array}\right\} A^{* \beta}+\left\{\begin{array}{c}
\sigma x \\
\beta
\end{array}\right\} A^{\alpha x} .
$$

Es ist dies der Ausdruck für die Erweiterung eines kontravarianten Tensors zweiten Ranges, der sich entsprechend auch für kontravariante Tensoren höheren und niedrigeren Ranges bilden läßt.

Wir merken an, daß sich auf analogem Wege auch die Erweiterung eines gemischten Tensors $A_{\mu}^{\alpha}$ bilden läßt:

$$
A_{\mu \sigma}^{\alpha}=\frac{\partial A_{\mu}^{\alpha}}{\partial x_{\sigma}}-\left\{\begin{array}{c}
\sigma \mu \\
\tau
\end{array}\right\} A_{\tau}^{\alpha}+\left\{\begin{array}{c}
\sigma \tau \\
\alpha
\end{array}\right\} A_{\mu}^{\tau} .
$$

Durch Verjüngung von (38) bezüglich der Indizes $\beta$ und $\sigma$ (innere Multiplikation mit $\delta_{\beta}^{\sigma}$ ) erhält man den kontravarianten Vierervektor

$$
A^{\alpha}=\frac{\partial A^{\alpha \beta}}{\partial x_{\beta}}+\left\{\begin{array}{c}
\beta x \\
\beta
\end{array}\right\} A^{\alpha x}+\left\{\begin{array}{c}
\beta x \\
\alpha
\end{array}\right\} A^{* \beta} .
$$

Wegen der Symmetrie von $\left\{\begin{array}{c}\beta_{a} \\ a\end{array}\right\}$ bezüglich der Indizes $\beta$ und $x$ verschwindet das dritte Glied der rechten Seite, falls $A^{\alpha \beta}$ ein antisymmetrischer Tensor ist, was wir annehmen wollen; das zweite Glied läßt sich gemäß (29a) umformen. Man erhält also

$$
A^{a}=\frac{1}{\sqrt{-g}} \frac{\partial\left(\sqrt{-g} A^{\alpha \beta}\right)}{\partial x_{\beta}} .
$$

Dies ist der Ausdruck der Divergenz eines kontravarianten Sechservektors.

Divergenz des gemischten Tensors zweiten Ranges. Bilden wir die Verjüngung von (39) bezüglich der Indizes $\alpha$ und $\sigma$, so erhalten wir mit Rücksicht auf (29a)

$$
\sqrt{-g} A_{\mu}=\frac{\partial\left(\sqrt{-g} A_{\mu}^{\sigma}\right)}{\partial \Sigma_{\sigma}}-\left\{\begin{array}{c}
\sigma \mu \\
\tau
\end{array}\right\} \sqrt{-g} A_{\tau}^{\sigma}
$$

Führt man im letzten Gliede den kontravarianten Tensor $A^{\rho^{\sigma}}=g^{e^{\tau}} A_{x}^{\sigma}$ ein, so nimmt es die Form an

$$
-\left[\begin{array}{c}
\sigma \mu \\
\rho
\end{array}\right] \sqrt{-g} A^{\rho^{\sigma}}
$$

Ist ferner der Tensor $A^{e^{\sigma}}$ ein symmetrischer, so reduziert sich dies auf

$$
-\frac{1}{2} \sqrt{-y} \frac{\partial g_{\varrho \sigma} \sigma}{\partial x_{\mu}} A^{\rho \sigma}
$$

Hätte man statt. $A^{\rho^{\sigma}}$ den ebenfalls symmetrischen kovarianten 
Tensor $A_{e \sigma}=g_{\rho a} g_{o \beta} A^{a \beta}$ eingeführt, so würde das letzte Glied vermöge (31) die Form

$$
\frac{1}{2} \sqrt{-g} \frac{\partial g^{\rho \sigma}}{\partial x_{\mu}} A_{e \sigma}
$$

annehmen. In dem betrachteten Symmetriefalle kann also (41) auch durch die beiden Formen

$$
\sqrt{-g} A_{\mu}=\frac{\partial\left(\sqrt{-g} A_{\mu}^{\sigma}\right)}{\partial x_{\sigma}}-\frac{1}{2} \frac{\partial g_{\rho} \rho}{\partial x_{\mu}} \sqrt{-g} A^{\rho}
$$

und

\section{Q. $(41 \mathrm{~b})$}

$$
\sqrt{-g} A_{\mu}=\frac{\partial\left(\sqrt{-g} A_{\mu}^{\sigma}\right)}{\partial x_{\sigma}}+\frac{1}{2} \frac{\partial g^{\rho \sigma}}{\partial x_{\mu}} \sqrt{-g} A_{e \sigma}
$$

ersetzt werden, von denen wir im folgenden Gebrauch zu machen haben.

\section{12. Der Riemann-Christoffelsche Tensor.}

Wir fragen nun nach denjenigen Tensoren, welche aus dem Fundamentaltensor der $g_{\mu}$ allein durch Differentiation gewonnen werden können. Die Antwort scheint zunächst auf der Hand zu liegen. Man setzt in (27) statt des beliebig gegebenen Tensors $A_{\mu v}$ den Fundamentaltensor der $g_{\mu v}$ ein und erhält dadurch einen neuen Tensor, nämlich die Erweiterung des Fundamentaltensors. Man überzeugt sich jedoch leicht, da $B$ diese letztere identisch verschwindet. Man gelangt jedoch auf folgendem Wege zum Ziel. Man setze in (27)

$$
A_{\mu \nu}=\frac{\partial A_{\mu}}{\partial x_{\nu}}-\left\{\begin{array}{c}
\mu \nu \\
\rho
\end{array}\right\} A_{e},
$$

d. h. die Erweiterung des Vierervektors $A_{\text {f }}$ ein. Dann erhält man (bei etwas geänderter Benennung der Indizes) den Tensor dritten Ranges

$$
\begin{aligned}
A_{\mu \sigma \tau} & =\frac{\partial^{2} A_{\mu}}{\partial x_{\sigma} \partial x_{\tau}} \\
\therefore & -\left\{\begin{array}{c}
\mu \sigma \\
\varrho
\end{array}\right\} \frac{\partial A_{\ell}}{\partial x_{\tau}}-\left\{\begin{array}{c}
\mu \tau \\
\varrho
\end{array}\right\} \frac{\partial A_{\ell}}{\partial x_{\sigma}}-\left\{\begin{array}{c}
\sigma x \\
\rho
\end{array}\right\} \frac{\partial A_{\mu}}{\partial x_{e}} \\
& +\left[-\frac{\partial}{\partial x_{\tau}}\left\{\begin{array}{c}
\mu \sigma \\
\varrho
\end{array}\right\}+\left\{\begin{array}{c}
\mu x \\
\alpha
\end{array}\right\}\left\{\begin{array}{c}
\alpha \sigma \\
\varrho
\end{array}\right\}+\left\{\begin{array}{c}
\sigma x \\
\alpha
\end{array}\right\}\left\{\begin{array}{c}
\alpha \mu \\
\rho
\end{array}\right\}\right] A_{e} .
\end{aligned}
$$

Dieser Ausdruck ladet zur Bildung des Tensors $A_{\mu \sigma \tau}-A_{\mu \text { to }}$ ein. Denn dabei heben sich folgende Terme des Ausdruckes für $A_{\mu \sim r}$ gegen solche von $A_{\mu \tau \sigma}$ weg: das erste Glied, das vierte

$$
-39-
$$

Glied, sowie das dem letzten Term in der eckigen Klammer entsprechende Glied; denn alle diese sind in $\sigma$ und $\tau$ symmetrisch. Gleiches gilt von der Summe des zweiten und dritten Gliedes. Wir erhalten also

(42)

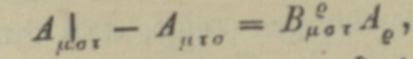

$$
\begin{aligned}
& \left\{\begin{aligned}
B_{\mu \sigma \tau}^{e}= & -\frac{\partial}{\partial x_{\tau}}\left\{\begin{array}{c}
\mu \sigma \\
\rho
\end{array}\right\}+\frac{\partial}{\partial x_{\sigma}}\left\{\begin{array}{c}
\mu \tau \\
\rho
\end{array}\right\} \\
& -\left\{\begin{array}{c}
\mu \sigma \\
\alpha
\end{array}\right\}\left\{\begin{array}{c}
\alpha \tau \\
\rho
\end{array}\right\}+\left\{\begin{array}{c}
\mu \tau \\
\alpha
\end{array}\right\}\left\{\begin{array}{c}
\alpha \sigma \\
e
\end{array}\right\} .
\end{aligned}\right.
\end{aligned}
$$

Wesentlich ist an diesem Resultat, $\mathrm{da} B$ auf der rechten Seite Werehr ihre Ableitungen aufvon (42) nur die $A_{e}$, aber nicht mehr inre treten. Aus dem Tensorcharakter von $A_{\mu \sigma \tau}-A_{\mu r \sigma}$ bindung damit, daß $A_{e}$ ein frei wählbarer $B_{\mu \sigma \text { ein Tensor }}^{e}$ folgt, vermöge der Resultate des $\$ 7$, da

ist (Riemann-Christoffelscher Tensor). Tensors liegt im

Die mathematische Bedeutung dieses Tensors liegt im folgenden. Wenn das Kontinuum so beschaffen ist, da $B$ es ein Koordinatensystem gibt, bezüglich dessen die $g_{j \nu}$ Konein Koordinatensystem gibt, bezalghich dessen dialt man statt des stanten sind, so verschwinden alle $R_{\mu \sigma r}^{e}$. Wahit man statt des ursprünglichen Koordinatensystems ein beliebiges neues, so ursprunglichen werden die auf letzteres bezogenen $g_{\mu \nu}$ nicht Konstantes ser mit sich, daß Der Tensorcharakter von $R_{\mu \sigma r}^{e}$ bringt es aber mit sich, das diese Komponenten auch in dem beliebig gewählten Bezugssystem sämtlich verschwinden. Das Verschwinden des Riemannschen Tensors ist also eine notwendige Bedingung da $B$ durch geeigmete Wahl des Bezugssystems die Konstanz

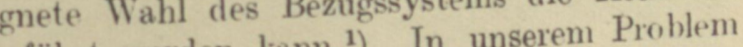
der $g_{\mu \nu}$ herbeigeführt werden kann. ${ }^{\text {) }}$ In unserem entspricht dies dem Falle, daß bei passender Wahl des Relaordinatensystems in endlichen Gebieten die spezielle Relativitätstheorie gilt.

Durch Verjüngung von (43) bezüglich der Indizes $\tau$ und $\varrho$ erhält man ten kovarianten Tensor zweiten Ranges

$$
\left\{\begin{array}{l}
B_{\mu v}=R_{\mu \nu}+S_{\mu v} \\
R_{\mu v}=-\frac{\partial}{\partial x_{\alpha}}\left\{\begin{array}{c}
\mu \nu \\
\alpha
\end{array}\right\}+\left\{\begin{array}{c}
\mu \alpha \\
\beta
\end{array}\right\}\left\{\begin{array}{c}
\nu \beta \\
\alpha
\end{array}\right\} \\
S_{\mu \nu}=\frac{\partial \lg \sqrt{-g}}{\partial x_{\mu} \partial x_{v}}-\left\{\begin{array}{c}
\mu \nu \\
\alpha
\end{array}\right\} \frac{\partial \lg \sqrt{-g}}{\partial x_{a}} .
\end{array}\right.
$$

Die eine hinreichende ist. 


$$
-40-
$$

Bemerkung über die Koordinatenwahl. Es ist sehon in $\$ 8$ im Anschluß an Gleichung (18a) bemerkt worden, da $B$ die Koordinatenwahl mit Vorteil so getroffen werden kann, daß $\sqrt{-g}=1$ wird. Ein Blick auf die in den beiden letzten Paragraphen erlangten Gleichungen zeigt, $\mathrm{da} B$ durch eine solche Wahl die Bildungsgesetze der Tensoren eine bedeutende Ver. einfachung erfahren. Besonders gilt dies für den soeben entwickelten Tensor $B_{u v}$, welcher in der darzulegenden Théorie eine fundamentale Rolle spielt. Die ins Auge gefaBte Speziali. sierung der Koordinatenwahl bringt nămlich das Verschwinden von $S_{\mu v}$ mit sich, so daB sich der Tensor $B_{\mu v}$ auf $R_{\mu \nu}$ reduziert.

Ich will deshalb im folgenden alle Beziehungen in der vereinfachten Form angeben, welehe die genannte Spezialisierung der Koordinatenwahl mit sieh bringt. Es ist dann ein Leichtes, auf die allgemein kovarianten Gleichungen zurückzugreifen, falls dies in einem speziellen Falle erwünscht erscheint.
C. Theorie des Gravitationsfeldes.

8 13. Bewegungsgleichung des materiellen Punktes im Gravitationsfeld.

Ansdruck für die Feldkomponenten der Gravitation.

Ein frei beweglicher, äußeren Kräften nicht unterworfener Körper bewegt sich nach der speziellen Relativitätstheorie geradlinig und gleichförmig. Dies gilt auch nach der allgemeinen Relativitätstheorie für einen Teil des vierdimensionalen Raumes, Relativitätstheor Koordinatensystem $K_{0}$ so wählbar und so gewählt ist, daß die $g_{\mu \nu}$ die in (4) gegebenen speziellen konstanten Werte haben.

Betrachten wir eben diese Bewegung von einem beliebig gewählten Koordinatensystem $K_{1}$ aus, so bewegt er sich von $K_{1}$ aus, beurteilt nach den Überlegungen des $\$ 2$ in einem Gravitationsfelde. Das Bewegungsgesetz mit Bezug auf $K_{1}$. ergibt sich leicht aus folgender Überlegung. Mit Bezug auf $K_{0}$ ist das Bewegungsgesetz eine vierdimensionale Gerade, also eine geodätische Linie. $\mathrm{Da}$ nun die geodätische Linie unabhängig vom Bezugssystem definiert ist, wird ihre Gleichung auch die Bewegungsgleichung des materiellen Punktes in bezug auf $K_{1}$ sein. Setzen wir

$$
\text { (45) } \quad \Gamma_{\mu \nu}^{\tau}=-\left\{\begin{array}{c}
\mu \nu \\
\tau
\end{array}\right\} \text {, }
$$

so lautet also die Gleichung der Punktbewegung in bezug auf $K_{1}$

$$
\text { (46) } \quad \frac{d^{2} x_{\tau}}{d s^{2}}=\Gamma_{\mu v}^{\tau} \frac{d x_{v}}{d s} \frac{d x_{v}}{d s} \text {. }
$$

Wir machen nun die sehr naheliegende Annahme, daß dieses allemein Bewegung des 


\section{$-42-$}

im Gravitationsfeld auch in dem Falle bestimmt, Punktes im Gravitationsfeld auch ien deziglich dessen in end. daß kein Bezugsita hichen Ratan die sperse Ab. Annahme sind wir um so berechtigter, als (46) nur erste Ab. leitungen der $g_{\mu \nu}$ enthălt, zwischen denen auch im Spezial. falle der Existenz von $K_{0}$ keine Beziehungen bestehen. ${ }^{1}$ )

Verschwinden die $\Gamma_{u v}^{\tau}$, so bewegt sich der Punkt geradinig und gleichförmig; diese Größen bedingen also die Ablinig und geichung der Bewegung von der Gleichförmigkeit. Sie sind die Komponenten des Gravitationsfeldes.

\$ 14. Die Feldgleichungen der Gravitation bel Abwesenheit von Materie.

Wir unterscheiden im folgenden zwischen „Gravitationsfeld" und "Materie", in dem Sinne, daß alles außer den Gravitationsfeld als "Materie" bezeichnet wird, also nicht nur die „Materie“ im üblichen Sinne, sondern auch das elektromagnetische Feld.

Unsere nächste Aufgabe ist es, die Feldgleichungen der Gravitation bei Abwésenheit von Materie aufsusuehen. Dabe verwenden wir wieder dieselbe Methode wie in vorigen Paragraphen bei der Aufstellung der Bewegungsgleichung des materiellen Punktes. Ein Spezialfall, in welchem die gesuchten Feldgleichungen jedenfalls erfüllt sein müssen, ist der der ursprünglichen Relativitätstheorie, in dem die $g_{\mu v}$ gewisse konstante Werte haben. Dies sei der Fall in einem gewissen endlichen Gebiete in bezug auf ein bestimmtes Koordinatensystem $K_{0}$. In bezug auf dies System verschwinden sămtliehe Komponenten $B^{\ell}$,des Riemannschen Tensors [Gleichung (43)] Diese verschwinden dann für das betrachtete Gebiet anch bezüglich jedes anderen Koordinatensystems.

Die gesuchten Gleichungen des materiefreien Gravitations. feldes müssen also jedenfalls erfüllt sein, wenn alle $B_{p e r}^{e}$ verschwinden. Aber diese Bedingung ist jedenfalls eine zu weit

1) Erst zwischen den zweiten (und ersten) Ableitungen bestehen gemä $\$ 12$ die Beziehungen $B C=0$. gehende. Denn es ist klar; daß z. B. das von einem Massenpunkte in seiner Umgebung erzeugte Gravitationsfeld sicherlich durch keine Wahl des Koordinatensystems „wegtransformiert", d. h. auf den Fall konstanter $g_{\mu \nu}$ transformiert werden kann.

Deshalb liegt es nahe, für das materiefreie Gravitationseld das Verschwinden des aus dem Tensor $B_{\mu \sigma \tau}^{e}$ abgeleiteten symmetrischen Tensors $B_{u v}$ zu verlangen. Man erhält so 10 Gleichungen für die 10 Größen $g_{\mu v}$, welche im speziellen erfüllt sind, wenn sämtliche $B_{\mu \sigma \tau}^{e}$ verschwinden. Diese Gleichungen lauten mit Rücksicht auf (44) bei der von uns getroffenen Wahl für das Koordinatensystem für das materiefreie Feld

(47)

$$
\left\{\begin{array}{c}
\frac{\partial \Gamma_{\mu \nu}^{a}}{\partial x_{\alpha}}+\Gamma_{\mu \beta}^{a} \Gamma_{v a}^{\beta}=0 \\
\sqrt{-g}=1 .
\end{array}\right.
$$

Es muß darauf hingewiesen werden, daß der Wahl dieser Gieich vinimum von Willkür anhaftet. Denn es Gleichungen der aus den gibt außer $B_{\mu \nu}$ keinen Tensor zweiten hanges, höheren als $g_{\mu \nu}$ und deren Ableitungen gebildet ist, keine horen ist. ${ }^{1}$ ) zweite Ableitungen enthält und in letzteren linear ist. ${ }^{1}$ )

$\mathrm{Da} B$ diese aus der Forderung der allgemeinen Relativität auf rein mathematischem Wege fließenden Gleichungen in Verbind 列 rung das Newtonsche Attraktionsgesetz, in zweiten (nach rung die Erklärung der von Leverrier entdeckten (rihel Anbringung der Störungskorrektionen übrigblen Ansicht von

bewegung des Merkur liefern, muß nach meiner Ansicht

\section{15. Hamiltonsche Funktion für das Gravitationsfeld,} Impulsenergiesatz.

Um the reldgleienter energiesatz entsprechen, ist es am bequer

Hamilton scher Form zu schreiben:

1) Eigentlich läßt sich dies nur von dem Tensor $B_{\mu \nu}+\lambda g_{\mu \nu}\left(g^{a \beta} B_{a \beta}\right)$

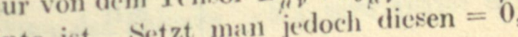
o kommt man wieder zu den Gilechungen $B_{\mu \nu}=0$. 


\section{(47a)}

$$
\left\{\begin{array}{c}
\delta\left\{\int H d \tau\right\}=0 \\
H=g^{\mu \nu} \Gamma_{\mu \beta}^{a} \Gamma_{v a}^{\beta} \\
\sqrt{-g}=1
\end{array}\right.
$$

Da iren des beDabei verschwinden ionsionalen Integrationsraumes.

Es ist zunächst zu zeigen, daß die Form (47a) den Gleichungen (47) äquivalent ist. Zu diesem Zweck betrachten wir $H$ als Funktion der $g^{\mu \nu}$ und der

$$
g_{\sigma}^{\mu v}\left(=\frac{\partial g^{\mu v}}{\partial x_{\sigma}}\right) \text {. }
$$

Dann ist zunächst

$$
\begin{aligned}
\delta H & =\Gamma_{\mu \beta}^{a} \Gamma_{v a}^{\beta} \delta g^{\mu \nu}+2 y^{\mu \nu} \Gamma_{\mu \beta}^{a} \delta \Gamma_{v a}^{\beta} \\
& =-\Gamma_{\mu \beta}^{a} \Gamma_{v a}^{\beta} \delta y^{\mu \nu}+2 \Gamma_{\mu \beta}^{a} \delta\left(g^{\mu \nu} \Gamma_{v a}^{\beta}\right) .
\end{aligned}
$$

Nun ist aber

$$
\delta\left(g^{\mu \nu} \Gamma_{v a}^{\beta}\right)=-\frac{1}{2} \delta\left[g^{\mu \nu} g^{\beta \lambda}\left(\frac{\partial g_{v \lambda}}{\partial x_{\alpha}}+\frac{\partial g_{a \lambda}}{\partial x_{r}}-\frac{\partial g_{a \nu}}{\partial x_{\lambda}}\right)\right]
$$

Die aus den beiden letzten Termen der runden Klammer hervorgehenden Terme sind von verschiedenem Vorzeichen und gehen auseinander (da die Benennung der Summationsindizes belanglos ist) durch Vertauschung der Indizes $\mu$ und $\beta$ hervor. Sie heben einander im Ausdruck für $\delta H$ weg, weil sie mit der bezüglich der Indizes $\mu$ und $\beta$ symmetrischen Größe $\Gamma_{\mu \beta}^{a}$ multipliziert werden. Es bleibt also nur das erste Glied der runden Klammer zu berücksichtigen, so da $\beta$ man mit Rücksicht auf (31) erhält

$$
\delta H=-\Gamma_{\mu \beta}^{a} \Gamma_{v a}^{\beta} \delta g^{\mu \nu}+I_{\mu \beta}^{a} \delta g_{a}^{\mu \beta} .
$$

Es ist also

(48)

$$
\left\{\begin{array}{l}
\frac{\partial H}{\partial g^{\alpha \nu}}=-I_{\mu \beta}^{a} \Gamma_{v a}^{\beta} \\
\frac{\partial H}{\partial y_{\sigma}^{\mu v}}=I_{\mu v}^{\sigma} .
\end{array}\right.
$$

$$
-45-
$$

Die Ausführung der Variation in (47a) ergibt zunächst das Gleichungssystem

$$
\frac{\partial}{\partial x_{a}}\left(\frac{\partial H}{\partial g_{a}^{\mu \nu}}\right)-\frac{\partial H}{\partial g^{\mu \nu}}=0
$$

welches wegen (48) mit (47) übereinstimmt, was zu beweisen war. - Multipliziert man $(47 \mathrm{~b})$ mit $g_{\sigma}^{\mu \nu}$, so erhält man, weil

und folglich

$$
\frac{\partial g_{a}^{\mu \nu}}{\partial x_{a}}=\frac{\partial g_{a}^{\mu v}}{\partial x_{a}}
$$

$$
g_{\sigma}^{\mu \nu} \frac{\partial}{\partial x_{a}}\left(\frac{\partial H}{\partial g_{a}^{\mu \nu}}\right)=\frac{\partial}{\partial x_{a}}\left(g_{\sigma}^{\mu \nu} \frac{\partial H}{\partial g_{a}^{\mu \nu}}\right)-\frac{\partial H}{\partial y_{a}^{\mu \nu}} \frac{\partial g_{a}^{\mu \nu}}{\partial x_{\sigma}}
$$

die Gleichung

$$
\frac{\partial}{\partial x_{a}}\left(g_{\sigma}^{\mu v} \frac{\partial H}{\partial g_{a}^{\mu \nu}}\right)-\frac{\partial H}{\partial x_{\sigma}}=0
$$

oder $^{1}$ )

$$
\left\{\begin{array}{c}
\frac{\partial t_{\sigma}^{a}}{\partial x_{a}}=0 \\
-2 x t_{\sigma}^{a}=g_{a}^{\mu \nu} \frac{\partial H}{\partial g_{a}^{\mu \nu}}-\delta_{\sigma}^{a} H
\end{array}\right.
$$

oder, wegen (48), der zweiten Gleichung (47) und (34)

(50) $\quad x t_{\sigma}^{a}=\frac{1}{2} \delta_{\sigma}^{a} g^{\mu \nu} \Gamma_{\mu \beta}^{\lambda} \Gamma_{v \alpha}^{\beta}-g^{\mu \nu} \Gamma_{\mu \beta}^{a} \Gamma_{v o}^{\beta}$.

Es ist zu beachten, daß $t_{\sigma}{ }^{a}$ kein Tensor ist; dagegen gilt (49) für alle Koordinatensysteme, für welche $\sqrt{-g}=1$ ist. (49) für alle Koordinatensystemes fungulses und Diese Gleichung drückt den Erhaltungssatz In der Tat liefert der Energie für das Gravitationsfeld aus. In dreidimensionales die Integration dieser Gleichung

(49a) $\frac{d}{d x_{4}}\left\{\int t_{\sigma}{ }^{4} d V\right\}=\int\left(t_{\sigma}{ }^{1} \alpha_{1}+t_{\sigma}{ }^{2} \alpha_{2}+t_{\sigma}{ }^{3} \alpha_{3}\right) d S$,

wichtungskosinus der nach innen gewobei $a_{1}, a_{2}, a_{3}$ der richteten Normale eines Flächenelementes der Begrenzes

1) Der Grund der Einführung des Faktors $-2 x$ wird später deutlich werden. 


$$
-46-
$$

von der (Größe $d S$ (im Sinne der euklidischen Geometrie) bedeuten. Man erkennt hierin den Ausdruck. der Erhaltungssätze in üblicher Fassung. Die Größen $t_{\sigma}{ }^{a}$ bezeichnen wir als die „Energiekomponenten“" des Gravitationsfeldes.

Ich will nun die Gleichungen (47) noch in einer dritten Form angeben, die einer lebendigen Erfassung unseres Gegenstandes besonders dienlich ist. Durch Multiplikation der Feldgleichungen (47) mit $g^{\nu \sigma}$ ergeben sich diese in der ,gemischten" Form. Beachtet man, daß

$$
g^{v \sigma} \frac{\partial \Gamma_{\mu \nu}^{\alpha}}{\partial x_{\alpha}}=\frac{\partial}{\partial x_{\alpha}}\left(g^{* \sigma} \Gamma_{\mu r}^{\alpha}\right)-\frac{\partial g^{\nu \sigma}}{\partial x_{\alpha}} \Gamma_{\mu v}^{\alpha},
$$

welche Größe wegen (34) gleich

$$
\frac{\partial}{\partial x_{\alpha}}\left(g^{r \sigma} \Gamma_{\mu v}^{a}\right)-g^{\gamma \beta} \Gamma_{a \beta}^{\sigma} \Gamma_{\mu r}^{a}-g^{\sigma \beta} \Gamma_{\beta \alpha}^{v} \Gamma_{\mu v}^{a},
$$

oder (nach geänderter Benennung der Summationsindizes) gleich

$$
\frac{\partial}{\partial x_{\alpha}}\left(g^{\alpha \beta} \Gamma_{\mu \beta}^{a}\right)-g^{m n} \Gamma_{m \beta}^{\sigma} \Gamma_{n \mu}^{\beta}-g^{r \sigma} \Gamma_{\mu \beta}^{a} \Gamma_{v a}^{\beta} .
$$

Das dritte Glied dieses Ausdrucks hebt sich weg gegen das aus dem zweiten Glied der Feldgleichungen (47) entstehende; an Stelle des zweiten Gliedes dieses Ausdruckes läßt sich nach Beziehung (50)

$$
\boldsymbol{x}\left(t_{\mu}{ }^{\sigma}-\frac{1}{2} \delta_{\mu}{ }^{\circ} t\right)
$$

setzen $\left(t=t_{a}{ }^{a}\right)$. Man erhält also an Stelle der Gleichungen (47)

$$
\left\{\begin{array}{c}
\frac{\partial}{\partial x_{\alpha}}\left(g^{\alpha \beta} \Gamma_{\mu \beta}^{a}\right)=-x\left(t_{\mu}{ }^{\sigma}-\frac{1}{2} \delta_{\mu}{ }^{\circ} t\right) \\
\sqrt{-g}=1
\end{array}\right.
$$

\section{16. Allgemeine Fassung der Feldgleichungen der Gravitation.}

Die im vorigen Paragraphen aufgestellten Feldgleichungen für materiefreie Räume sind mit der Feldgleichung

$$
\Delta p=0
$$

der Newtonschen Theorie zu vergleichen. Wir haben die Gleichungen aufzusuchen, welche der Poissonschen Gleichung

$$
\Delta \varphi=4 \pi x \underline{\varphi}
$$

entspricht, wobei $\varrho$ die Dichte der Materie bedeutet.

$$
-47-
$$

Die spezielle Relativitätstheorie hat zu dem Ergebnis geführt, da $\beta$ die träge Masse nichts anderes ist als Energie, welche ihren vollständigen mathematischen Ausdruck in einem symmetrischen Tensor zweiten Ranges, dem Energietensor, findet. Wir werden daher auch in der allgemeinen Relativitäts theorie einen Energietensor der Materie $T^{a}$ einzuführen haben, der wie die Energiekomponenten $t_{\sigma}{ }^{a}$ [Gleichungen.(49) und (50)] des Gravitationsfeldes gemischten Charakter haben wird, aber zu einem symmetrischen kovarianten Tensor gehören wird ${ }^{1}$ ).

Wie dieser Energietensor (entsprechend der Dichte $\varrho$ in der Poissonschen Gleichung) in die Feldgleichungen der Gravitation einzuführen ist, lehrt das Gleichungssystem (51). Betrachtet man nämlich ein vollständiges System (z. B. das Sonnensystem), so wird die Gesamtmasse des Systems, also auch seine gesamte gravitierende Wirkung, von der Gesamtenergie des Systems, also von der ponderablen und Gravitationsenergie zusammen, abhängen. Dies wird sich dadurch ausdrücken lassen, da $B$ man in (51) an Stelle der Energiekomponenten $t_{\mu}{ }^{\circ}$ des Gravitationsfeldes allein die Summen $t_{\mu}{ }^{\sigma}+T_{\mu}{ }^{\sigma}$ der Energiekomponenten von Materie und Gravi. tationsfeld einführt. Man erhält so statt (51) die Tensorgleichung

$$
\left\{\begin{array}{c}
\frac{\partial}{\partial x_{\alpha}}\left(g^{\sigma \beta} \Gamma_{\mu \beta}^{a}\right)=-x\left[\left(t_{\mu}{ }^{\sigma}+T_{\mu}{ }^{\sigma}\right)-\frac{1}{2} \delta_{\mu}{ }^{\sigma}(t+T)\right] \\
\sqrt{-g}=1
\end{array}\right.
$$

wobei $T=T_{\mu}^{\mu}$ gesetzt ist (Lauescher Skalar). Dies sind die gesuchten allgemeinen Feldgleichungen der Gravitation in gemischter Folm. An Stelle von (47) ergibt sich daraus rückwärts das System

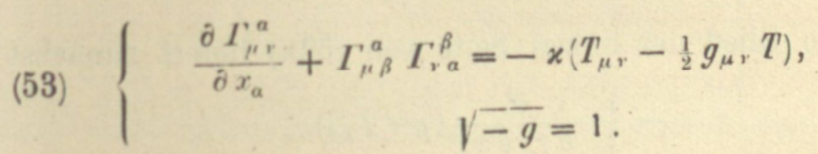

Es muß zugegeben werden, daß diese Einführung des Energietensors der Materie durch das Relativitätspostulat allein nicht gerechtfertigt wird; deshalb haben wir sie im 1) $g_{0} T_{o}^{a}=T_{o}$ und $g^{\alpha \beta} T_{a}^{\alpha}=T^{a \beta}$ sollen symmetrische Tensoren sein. 
Af rigen aus der Forderung abgeleitet, daß die Energie des Gravitationsfeldes in gleicher Weise gravitierend wirken soll, wie jegliche Energie anderer Art. Der stärkste Grund für die Wahl der vorstehenden Gleichungen liegt aber darin, daß sie zur Folge haben, daß für die Komponenten der Totalenergie Erhaltungsgleichungen (des Impulses und der Energie) gelten, welche den Gleichungen (49) und (49a) genau entsprechen. Dies soll im folgenden dargetan werden.

\section{17. Die Erhaltungssätze im allgemeinen Falle.}

Die Gleichung (52) ist leicht so umzuformen, daß auf der rechten Seite das zweite Glied wegfällt. Man verjünge (52) nach den Indizes $\mu$ und $\sigma$ und subtrahiere die so erhaltene, mit $\frac{1}{2} \delta_{\mu}{ }^{\circ}$ multiplizierte Gleichung von (52). Es ergibt sich

(52a) $\frac{\partial}{\partial x_{\alpha}}\left(g^{\sigma \beta} \Gamma_{\mu \beta}^{a}-\frac{1}{2} \partial_{\mu}{ }^{\sigma} g^{\lambda \beta} \Gamma_{\lambda \beta}^{a}\right)=-x\left(t_{\mu}{ }^{\sigma}+T_{\mu}{ }^{\sigma}\right)$.

An dieser Gleichung bilden wir die Operation $\partial / \partial x_{\sigma}$. Es ist

$$
\begin{aligned}
\frac{\partial^{2}}{\partial x_{a} \partial x_{\sigma}} & \left(g^{\sigma \beta} \Gamma_{\mu \beta}^{a}\right) \\
& =-\frac{1}{2} \frac{\dot{\theta}^{2}}{\partial x_{a} \partial x_{\sigma}}\left[g^{\sigma \beta} g^{\alpha \lambda}\left(\frac{\partial g_{\mu \lambda}}{\partial x_{\beta}}+\frac{\partial g_{\beta \lambda}}{\partial x_{\mu}}-\frac{\partial g_{\mu \beta}}{\partial x_{\lambda}}\right)\right] .
\end{aligned}
$$

Das erste und das dritte Glied der runden Klammer liefern Beiträge, die einander wegheben, wie man erkennt, wenn man im Beitrage des dritten Gliedes die Summationsindizes $\alpha$ und $\sigma$ einerseits, $\beta$ und $\lambda$ andererseits vertauscht. Das zweite Glied läßt sich nach (31) umformen, so daß man erhält

$$
\text { (54) } \quad \frac{\partial^{2}}{\partial x_{\alpha} \partial x_{\sigma}}\left(g^{\sigma \beta} I_{\mu \beta}^{a}\right)=\frac{1}{2} \frac{\partial^{3} g^{\alpha \beta}}{\partial x_{\alpha} \partial x_{\beta} \partial x_{\mu}} \text {. }
$$

Das zweite Glied der linken Seite von (52a) liefert zunächst

$$
-\frac{1}{2} \frac{\partial^{2}}{\partial x_{\alpha} \partial x_{\mu}}\left(g^{\lambda \beta} \Gamma_{\lambda \beta}^{a}\right)
$$

oder

$$
\frac{1}{4} \frac{\partial^{2}}{\partial x_{a} \partial x_{\mu}}\left[g^{\lambda \beta} g^{a \delta}\left(\frac{\partial g_{\partial \lambda}}{\partial x_{\beta}}+\frac{\partial g_{\delta \beta}}{\partial x_{\lambda}}-\frac{\partial g_{\lambda \beta}}{\partial x_{\delta}}\right)\right] \text {. }
$$

Das vom letzten Glied der runden Klammer herrührende Glied verschwindet wegen (29) bei der von uns getroffenen
Koordinatenwahl. Die beiden anderen lassen sich zusammenfassen und liefern wegen (31) zusammen

$$
-\frac{1}{2} \frac{\hat{\sigma}^{\mathrm{q}} g^{\alpha \beta}}{\partial x_{a} \partial x_{\beta} \partial x_{\mu}}
$$

so daß mit Rücksicht auf (54) die Identität

(55)

$$
\frac{\partial^{2}}{\partial x_{\alpha} \partial x_{\sigma}}\left(g^{\sigma \beta} \Gamma_{\mu \beta}^{a}-\frac{1}{2} \delta_{\mu}{ }^{\sigma} g^{\lambda \beta} \Gamma_{\lambda \beta}^{a}\right) \equiv 0
$$

besteht. Aus (55) und (52a) folgt

(56)

$$
\frac{\partial\left(t_{\mu^{\sigma}}+T_{\mu^{\sigma}}\right)}{\partial x_{\sigma}}=0 .
$$

Aus unseren Feldgleichungen der Gravitation geht also hervor, daß den Erhaltungssätzen des Impulses und der Energie Genüge geleistet ist. Man sieht dies am einfachsten nach der Betrachtung ein, die zu Gleichung (49a) führt; nur hat man hier an Stelle der Energiekomponenten $t_{\mu}{ }^{\sigma}$ des Gravitationsfeldes die Gesamtenergiekomponenten von "Materie und Gravitationsfeld einzuführen.

\& 18. Der Impulsenergiesatz für die Materie als Folge der Feldgleichungen.

Multipliziert man (53) mit $\partial g^{\mu \nu} / \partial x_{\sigma}$, so erhält man auf dem in $\S 15$ eingeschlagenen Wege mit Rücksicht auf das Verschwinden von

$$
g_{\mu \nu} \frac{\partial g^{\mu \nu}}{\partial x_{\sigma}}
$$

die Gleichung

$$
\frac{\partial t_{\sigma} a}{\partial x_{a}}+\frac{1}{2} \frac{\partial g^{\mu \nu}}{\partial x_{\sigma}} T_{\mu \nu}=0
$$

oder mit Rücksicht auf (56)

$$
\frac{\partial T_{g}^{a}}{\partial x_{a}}+\frac{1}{2} \frac{\partial g^{\mu \nu}}{\partial x_{\sigma}} T_{\mu \nu}=0 .
$$

Fin Vergleich mit ( $41 \mathrm{~b})$ zeigt, da $\beta$ diese Gleichung bei der getroffenen Wahl für das Koordinatensystem nichts anderes aussagt als das Verschwinden der Divergenz des Tensors der Energiekomponenten der Materie. Physikalisch zeigt das Aufreten daß für die Materie allein Erhaltungssätze des Impulses und der Energie im eigentEinstein. 
lichen Sinne nicht, bzw. nur dann gelten, wenn die $g^{\mu r}$ konstant sind, d. h. wenn die Feldstärken der Gravitation verschwinden. Dies zweite Glied ist ein Ausdruck für Impuls bzw. Energie, welche pro Volumen und Zeiteinheit rom Gravitationsfelde auf die Materie übertragen werden. Dies tritt noch klarer hervor, wenn man statt (57) im Sinne von (41) schreibt

\section{(57a)}

$$
\frac{\partial T_{\sigma}{ }^{a}}{\partial x_{\alpha}}=-I_{\sigma \beta}^{a} T_{a}^{\beta}
$$

Die rechte Seite drückt die energetische Einwirkung des Gravitationsfeldes auf die Materie aus.

Die Feldgleichungen der Gravitation enthalten also gleichzeitig vier Bedingungen, welchen der materielle Vorgang zu genügen hat. Sie liefern die Gleichungen des materiellen Vorvollständig, wenn letzterer durch vier voneinander unabhängige Differentialgleichungen charakterisierbar ist. ${ }^{1}$ )

1) Vgl, hierüber D. Hilbert, Nachr. d. K. Gesellsch. d. Wiss. zu Göttingen, Math.-phys. Klasse. p. 3. 1915. 


$$
T^{\alpha \beta}=-g^{\alpha \beta} p+\varrho \frac{d x_{a}}{d s} \frac{d x_{\beta}}{d s}
$$

sei der kontravariante Energietensor der Flüssigkeit. Zu ihm gehört der kovariante Tensor

(58a)

$$
T_{\mu \nu}=-g_{\mu \nu} p+g_{\mu a} \frac{d x_{a}}{d s} g_{\mu \beta} \frac{d x_{\beta}}{d s} \varrho,
$$

sowie der gemischte Tensor ${ }^{1}$ )

$$
T_{\sigma}{ }^{a}=-\delta_{\sigma}{ }^{a} p+g_{\sigma \beta} \frac{d x_{\beta}}{d s} \frac{d x_{a}}{d s} \varrho .
$$

Setzt man die rechte Seite von $(58 \mathrm{~b})$ in (57a) ein, so erhält man die Eulerschen hydrodynamischen Gleichungen der allgememen Relativitätstheorie. Diese lösen das Bewegungsproblem im Prinzip vollständig; denn die vier Gleichungen (57a) zusammen mit der gegebenen Gleichung zwischen $p$ und $\varrho$ und der Gleichung

$$
g_{a p} \frac{d x_{a}}{d s} \frac{d x_{\beta}}{d s}=1
$$

genügen bei gegebenen $g_{a \beta}$ zur Bestimmung der 6 Unbekannten

$$
p, \rho, \frac{d x_{1}}{d s}, \frac{d x_{2}}{d s}, \frac{d x_{3}}{d s}, \frac{d x_{4}}{d s} .
$$

Sind auch die $g_{\mu \nu}$ unbekannt, so kommen hierzu noch die Gleichungen (53). Dies sind 11 Gleichungen zur Bestimmung der 10 Funktionen $g_{\mu \nu}$, so daß diese überbestimmt scheinen. Es ist indessen zu beachten, daß die Gleichungen (57a) in den Gleichungen (53) bereits enthalten sind, so daß letztere nur mehr 7 unabhöngige Gleichungen repräsentieren. Diese Unbestimmtheit hat ihren guten Grund darin, daß die weitgehende Freiheit in der Wahl der Koordinaten es mit sich bringt, da $\beta$ das Problem mathematisch in solchem Grade unbestimmt bleibt, daß drei der Raumfunktionen beliebig gewählt werden können. ${ }^{2}$ )

1) Für einen mitbewegten Beobachter, der im unendlich Kleinen ein Bezugssystem im Sinne der speziellen Relativitätstheorie benutzt, st die Energiedichte $T_{4}{ }^{4}$ gleich $\varrho-p$. Hierin liegt die Definition von $\rho^{\circ}$ Es ist also $Q$ nicht konstant für eine inkompressible Flüssigkeit.

2) Bei Verzicht auf die Koordinatenwahl gemäß $g=-1$ blieben vier Raumfunktionen frei wählbar, entsprechend den vier willkürlichen Funktionen, über die man bei der Koordinatenwahl frei verfügen kann.

\section{\$20. Maxw ellsche elektromagnetische Feldgleichungen} für das Vakuum.

Es seien $\varphi_{2}$ die Komponenten eines kovarianten Vierervektors, des Vierervektors des elektromagnetischen Potentials. Aus ihnen bilden wir gemäß (36) die Komponenten $F$ des kovarianten Sechservektors des elektromagnetischen Feldes gemä $ß$ dem Gleichungssystem

$$
F_{\varrho \sigma}=\frac{\partial \varphi_{\varrho}}{\partial x_{\sigma}}-\frac{\partial q_{\sigma}}{\partial x_{\varrho}} .
$$

Aus (59) folgt, daß das Gleichungssystem

$$
\frac{\partial F_{\varrho \sigma}}{\partial x_{\tau}}+\frac{\partial F_{\sigma \tau}}{\partial x_{\varrho}}+\frac{\partial F_{\tau}}{\partial x_{\varrho}}=0
$$

erfüllt ist, dessen linke Seite gemä $B$ (37) ein antisymmetrischer Tensor dritten Ranges ist. Das System (60) enthält also im wesentlichen 4 Gleichungen, die ausgeschrieben wie folgt lauten:

$$
\left\{\begin{array}{l}
\frac{\partial F_{23}}{\partial x_{4}}+\frac{\partial F_{34}}{\partial x_{2}}+\frac{\partial F_{42}}{\partial x_{3}}=0 \\
\frac{\partial F_{34}}{\partial x_{1}}+\frac{\partial F_{41}}{\partial x_{3}}+\frac{\partial F_{13}}{\partial x_{4}}=0 \\
\frac{\partial F_{41}}{\partial x_{2}}+\frac{\partial F_{12}}{\partial x_{4}}+\frac{\partial F_{74}}{\partial x_{1}}=0 \\
\frac{\partial F_{12}}{\partial x_{3}}+\frac{\partial F_{23}}{\partial x_{1}}+\frac{\partial F_{31}}{\partial x_{2}}=0
\end{array}\right.
$$

Dieses Gleichungssystem entspricht dem zweiten Gleichungssystem Maxwells. Man erkennt dies sofort, indem man setzt

$$
\begin{cases}F_{23}=\mathfrak{h}_{x} & F_{14}=\mathfrak{e}_{x} \\ F_{31}=\mathfrak{h}_{y} & F_{24}=e_{y} \\ F_{12}=\mathfrak{h}_{z} & F_{34}=e_{z} .\end{cases}
$$

Dann kann man statt $(60 a)$ in üblicher Schreibweise der dreidimensionalen Vektoranalyse setzen

$$
\begin{gathered}
\frac{\partial \mathfrak{h}}{\partial t}+\operatorname{rot} e=0 \\
\operatorname{div} \mathfrak{h}=0 .
\end{gathered}
$$


Das erste Maxwellsche System erhalten wir durch Verallgemeinerung der von Minkowski angegebenen Form. Wir führen den zu $F_{a \beta}$ gehörigen kontravarianten Sechservektor (62)

$$
F^{\mu \nu}=g^{\mu a} q^{\nu \beta} F_{a \beta}
$$

ein sowie den kontravarianten Vierervektor $J^{\mu}$ der elektrischen Vakuumstromdichte; dann kann man das mit Rücksicht auf (40) gegenüber beliebigen Substitutionen von der Determinante 1 (gemä $\beta$ der von uns getroffenen Koordinatenwahl) invariante Gleichungssystem ansetzen:

$$
\frac{\partial F^{\mu \nu}}{\partial x_{\nu}}=J^{\mu}
$$

Setzt man nämlich

$$
\begin{cases}F^{23}=\mathfrak{h}_{x}^{\prime} & F^{14}=-\mathrm{e}_{x}^{\prime} \\ F^{31}=\mathfrak{h}_{y}^{\prime} & F^{24}=-\mathfrak{e}_{y}^{\prime} \\ F^{12}=\mathfrak{h}_{y}^{\prime} & F^{34}=-\mathfrak{e}_{y}^{\prime},\end{cases}
$$

welche Größen im Spezialfall der speziellen Relativitätstheorie den Größen $h_{x} \ldots e_{z}$ gleich sind, und außerdem

$$
J^{1}=\mathrm{i}_{x}, \quad J^{2}=\mathrm{i}_{y}, \quad J^{3}=\mathrm{i}_{z}, \quad J^{4}=\varrho,
$$

so erhält man an Stelle von $(63)$

$$
\left\{\begin{array}{c}
\operatorname{rot} \mathfrak{h}^{\prime}-\frac{\partial \mathrm{e}^{\prime}}{\partial t}=\mathrm{i} \\
\operatorname{div} \mathrm{e}^{\prime}=0
\end{array}\right.
$$

Die Gleichungen (60), (62) und (63) bilden also die Verallgemeinerung der Maxwellschen Feldgleichungen des Vakuums bei der von uns bezüglich der Koordinatenwahl getroffenen Festsetzung.

Die Energiekomponenten des elektromagnetischen Feldes. Wir bilden das innere Produkt

\section{(65)}

$$
\boldsymbol{x}_{\sigma}=F_{\sigma \mu} J^{\mu} .
$$

sine Komponenten lauten gemäß,(61) in dreidimensionaler Schreibwe ise

$$
\left\{\begin{array}{l}
x_{1}=\rho e_{x}+[\mathrm{i}, \mathfrak{h}]_{x} \\
x_{\mathrm{s}}=-(\mathrm{i}, \mathrm{e})
\end{array}\right.
$$

Es ist $\boldsymbol{x}_{0}$ ein kovarianter Vierervelitor, dessen Komponenten gleich sind dem negativen Impuls bzw. der Energie, welche pro Zeit- und Volumeinheit auf das elektromagnetische Feld von den elektrischen Massen übertragen werden. Sind die elektrischen Massen frei, d. h. unter dem alleinigen Einfluß des elektromagnetischen Feldes, so wird der kovariante Vierervektor $\boldsymbol{x}_{\boldsymbol{\sigma}}$ verschwinden.

Um die Energiekomponenten $T_{\sigma}{ }^{v}$ des elektromagnetischen Feldes zu erhalten, brauchen wir nur der Gleichung $\boldsymbol{x}_{\sigma}=0$ die Gestalt der Gleichung (57) zu geben. Aus (63) und (65) ergibt sich zunächst

$$
\boldsymbol{x}_{\sigma}=F_{\sigma \mu} \frac{\partial F^{\mu \nu}}{\partial x_{\nu}}=\frac{\partial}{\partial x_{\nu}}\left(F_{\sigma \mu} F^{\mu \nu}\right)-F^{\mu \nu} \frac{\partial F_{\sigma \mu}}{\partial x_{\nu}} .
$$

Das zweite Glied der rechten Seite gestattet vermöge (60) die Umformung

$$
F^{\mu \nu} \frac{\partial F_{\sigma \mu}}{\partial x_{\nu}}=-\frac{1}{2} F^{\mu \nu} \frac{\partial F_{\mu \nu}}{\partial x_{\sigma}}=-\frac{1}{2} g^{\mu \alpha} g^{\nu \beta} F_{\alpha \beta} \frac{\partial F_{\mu \nu}}{\partial x_{\sigma}},
$$

welch letzterer Ausdruck aus Symmetriegründen auch in der Form

$$
-\frac{1}{4}\left[g^{\mu a} g^{\nu \beta} F_{\alpha \beta} \frac{\partial F_{\mu \nu}}{\partial x_{\sigma}}+g^{\mu a} g^{v \beta^{\beta}} \frac{\partial F_{a \beta}}{\partial x_{\sigma}} F_{\mu v}\right]
$$

geschrieben werden kann. Dafür aber läßt sich setzen

$$
-\frac{1}{4} \frac{\partial}{\partial x_{\sigma}}\left(g^{\mu a} g^{\nu \beta} F_{\alpha \beta} F_{\mu \nu}\right)+\frac{1}{4} F_{\alpha \beta} F_{\mu \nu} \frac{\partial}{\partial x_{\sigma}}\left(g^{\mu a} g^{\nu \beta}\right) \text {. }
$$

Das erste dieser Glieder lautet in kürzerer Schreibweise

$$
-\frac{1}{4} \frac{\partial}{\partial x_{\sigma}}\left(F^{\mu \nu} F_{\mu \nu}\right),
$$

das zweite ergibt nach Ausführung der Differentiation nach einiger Umformung

$$
-\frac{1}{2} F^{\mu \tau} F_{\mu \nu} g^{\nu} e \frac{\partial g_{\sigma x}}{\partial x_{\sigma}} .
$$

Nimmt man alle drei berechneten Glieder zusammen, so erhält man die Relation

$$
x_{o}=\frac{\partial T_{o}^{\nu}}{\partial x_{\nu}}-\frac{1}{2} g^{t \mu} \frac{\partial g_{\mu \nu}}{\partial x_{o}} T_{\tau}^{\nu},
$$

wobej

$$
T_{o}^{v}=-F_{a a} F^{v a}+\frac{1}{4} \delta_{o}{ }^{v} F_{a \beta} F^{a \beta} .
$$


Die Gleichung (66) ist für verschwindendes $\boldsymbol{x}_{\sigma}$ wegen (30) mit (57) bzw. (57a) gleichwertig. Es sind also die $T_{\sigma}{ }^{v}$ die Energiekomponenten des elektromagnetischen Feldes. Mit Hilfe von (61) und (64) zeigt man leicht, daß diese Energiekomponenten des elektromagnetischen Feldes im Falle der speziellen Relativitätstheorie die wohlbekannten MaxwellPointingschen Ausdrücke ergeben.

Wir haben nun die allgemeinsten Gesetze abgeleitet, welchen das Gravitationsfeld und die Materie genügen, indem wir uns konsequent eines Koordinatensystems bedienten, für welches $\sqrt{-g}=1$ wird. Wir erzielten dadurch eine erhebliche Vereinfachung der Formeln und Rechnungen, ohne daß wir auf die Forderung der allgemeinen Kovarianz verzichtet hätten: denn wir fanden unsere Gleichungen durch Spezialisierung des Koordinatensystems aus allgemein kovarianten Gleichungen.

Immerhin ist die Frage nicht ohne formales Interesse, ob bei entsprechend verallgemeinerter Definition der Energiekomponenten des Gravitationsfeldes und der Materie auch ohne Spezialisierung des Koordinatensystems Erhaltungssätze von der Gestalt der Gleichung (56) sowie Feldgleichungen der Gravitation von der Art der Gleichungen (52) bzw. (52a) gelten, derart, daß links eine Divergenz (im gewöhnlichen Sinne), rechts die Summe der Energiekomponenten der Materie und der Gravitation steht. Ich habe gefunden, in der Tat der Fall ist. Doch glaube ich, daß sich eine Mitteilung meiner ziemlich umfangreichen Betrachtungen über diesen Gegenstand nicht lohnen würde, da doch etwas sachlich Neues dabei nicht herauskommt.

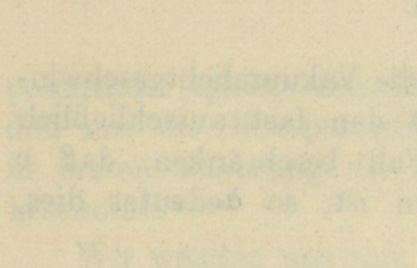

\section{E. \& 21. Newtons Theorie als erste Näherung.}

Wie schon mehrfach erwähnt, ist die spezielle Relativitätstheorie als Spezialfall der allgemeinen dadurch charakterisiert, daß die $g_{\mu \nu}$ die konstanten Werte (4) haben. Dies bedeutet nach dem Vorherigen eine völlige Vernachlässigung der Gravitationswirkungen. Eine der Wirklichkeit näher liegende Approximation erhalten wir, indem wir den Fall betrachten, da $\beta$ die $g_{\mu \nu}$ von den Werten (4) nur um (gegen 1) kleine Größen abweichen, wobei wir kleine Größen zweiten und höheren Grades vernachlässigen. (Erster Gesichtspunkt der Approximation.)

Ferner soll angenommen werden, da $\beta$ in dem betrachteten zeiträumlichen Gebiete die $g_{\mu \nu}$ im räumlich Unendlichen bei passender Wahl der Koordinaten den Werten (4) zustreben; d. h. wir betrachten Gravitationsfelder, welche als ausschließlich durch im Endlichen befindliche Materie erzeugt betrachtet werden können.

Man könnte annehmen, daß diese Vernachlässigungen auf Newtons Theorie hinführen müßten. Indessen bedarf es hierfür noch der approximativen Behandlung der Grundgleichungen nach einem zweiten Gesichtspunkte. Wir fassen die Bewegung eines Massenpunktes gemäß den Gleichungen (46) ins Auge. Im Falle der speziellen Relativitätstheorie können die Komponenten

$$
\frac{d x_{1}}{d s}, \frac{d x_{2}}{d s}, \frac{d x_{3}}{d s}
$$

beliebige Werte annehmen; dies bedeutet, daß beliebige Geschwindigkeiten

$$
v=\sqrt{\frac{d x_{1}^{2}}{d x_{4}}+\frac{d x_{9}^{2}}{d x_{4}}+\frac{d x_{3}{ }^{2}}{d x_{4}}}
$$


auftreten können, die kleiner sind als die Vakuumlichtgeschwindigkeit $(v<1)$. Will man sich auf den fast ausschließlich der Erfahrung sich darbietenden Fall beschränken, daß $v$ gegen die Lichtgeschwindigkeit klein ist, so bedeutet dies, daß die Komponenten

$$
\frac{d x_{1}}{d s}, \frac{d x_{2}}{d s}, \frac{d x_{3}}{d s}
$$

als kleine Größen zu behandeln sind, während $d x_{4} / d s$ bis auf Größen zweiter Ordnung gleich 1 ist (zweiter Gesichtspunkt der Approximation).

Nun beachten wir, daß nach dem ersten Gesichtspunkte der Approximation die Größen $\Gamma_{\mu \nu}^{\tau}$ alle kleine Größen mindestens erster Ordnung sind. Ein Blick auf (46) lehrt also, da B in dieser Gleichung nach dem zweiten Gesichtspunkt der Approximation nur Glieder $\mathrm{zu}$ berücksichtigen sind, für welche $\mu=v=4$ ist. Bei Beschränkung auf Glieder niedrigster Ordnung erhält man an Stelle von (46) zunächst die Gleichungen

$$
\frac{d^{2} x_{\tau}}{d t^{2}}=\Gamma_{44}^{2}
$$

wobei $d s=d x_{4}=d t$ gesetzt ist, oder unter Beschränkung auf Glieder, die nach dem ersten Gesichtspunkte der Approximation erster Ordnung sind:

$$
\begin{aligned}
& \frac{d^{2} x_{\tau}}{d t^{2}}=\left[\begin{array}{c}
44 \\
\tau
\end{array}\right](\tau=1,2,3) \\
& \frac{d^{2} x_{4}}{d t^{2}}=-\left[\begin{array}{c}
44 \\
4
\end{array}\right] .
\end{aligned}
$$

Setzt man auBerdem roraus, da $\beta$ das Gravitationsfeld ein quasi statisches sei, indem man sich auf den Fall beschränkt, daß die das Graritationsfeld erzeugende Materie nur langsam (im Vergleich mit der Fortpflanzungsgeschwindigkeit des Lichtes) bewegt ist, so kann man auf der rechten Seite Ableitungen nach der Zeit neben solchen nach den örtlichen Koordinaten vernachlässigen, so daß man erhält

$$
\frac{d^{2} x_{\tau}}{d t^{2}}=-\frac{1}{2} \frac{\partial g_{44}}{\partial x_{\tau}}(\tau=1,2,3) .
$$

Dies ist die Bewegungsgleichung: des materiellen Punktes nach Newtons Theorie, wobei $g_{44} / 2$ die Rolle des Gravitations- potentiales spieit. Das Merkwürdige an diesem Resultat ist, daß nur die Komponente $g_{44}$ des Fundamentaltensors allein in erster Näherung die Bewegung des materiellen Punktes bestimmt.

Wir wenden uns nun zu den Feldgleichungen (53). Dabei ist $\mathrm{zu}$ berücksichtigen, daß der Energietensor der "Materie“ fast ausschließlich durch die Dichte $\varrho$ der Materie im engeren Sinne bestimmt wird, d. h. durch das zweite Glied der rechten Seite von (58) [bzw. (58a) oder (58b)]. Bildet man die uns interessierende Näherung, so verschwinden alle Komponenten bis auf die Komponente

$$
T_{44}=\varrho=T .
$$

Auf der linken Seite von (53) ist das zweite Glied klein von zweiter Ordnung; das erste liefert in der uns interessierenden Näherung

$$
+\frac{\partial}{\partial x_{1}}\left[\begin{array}{c}
\mu \nu \\
1
\end{array}\right]+\frac{\partial}{\partial x_{2}}\left[\begin{array}{c}
\mu \nu \\
2
\end{array}\right]+\frac{\partial}{\partial x_{3}}\left[\begin{array}{c}
\mu \nu \\
3
\end{array}\right]-\frac{\partial}{\partial x_{4}}\left[\begin{array}{c}
\mu \nu \\
4
\end{array}\right]
$$

Dies liefert für $\mu=\nu=4$ bei Weglassung von nach der Zeit differenzierten Gliedern

$$
-\frac{1}{2}\left(\frac{\partial^{2} g_{44}}{\partial x_{1}{ }^{2}}+\frac{\partial^{2} g_{44}}{\partial x_{2}{ }^{2}}+\frac{\partial^{2} g_{44}}{\partial x_{3}{ }^{2}}\right)=-\frac{1}{2} \Delta g_{44} .
$$

Die letzte der Gleichungen (53) liefert also

$$
\Delta g_{44}=x \varphi \text {. }
$$

Die Gleichungen (67) und (68) zusammen sind äquivalent dem Newtonschen Gravitationsgesetz.

Für das Gravitationspotential ergibt sich nach (67) und (68) der Ausdruck '

$$
-\frac{x}{8 \pi} \int \frac{\varrho d x}{r}
$$

während Newtons Theorie bei der von uns gewählten Zeit. einheit

$$
-\frac{K}{c^{2}} \int \frac{\rho d \tau}{r}
$$

ergibt, wobei $K$ die gewöhnlich als Gravitationskonstante 
bezeichnete Konstante $6,7 \cdot 10^{-8}$ bedeutet. Durch Vergleich ergibt sich

$$
x=\frac{8 \pi K}{c^{2}}=1,87 \cdot 10^{-27}
$$

\$ 22. Verhalten von Maßstäben und Uhren im statischen Gravitationsfelde. Krümmung der Lichtstrahlen. Perihelbewegung der Planetenbahnen.

Um die Newton sche Theorie als erste Näherung zu erhalten, brauchten wir von den 10 Komponenten des Gravitationspotentials $g_{\mu \nu}$ nur $g_{44}$ zu berechnen, da nur diese Komponente in die erste Näherung (67) der Bewegungsgleichung des materiellen Punktes im Gravitationsfelde eingeht. sieht indessen schon daraus, daB noch andere Komponenten der $g_{\mu \nu}$ von den in (4) angegebenen Werten in erster Näherung abweichen müssen, daß letzteres durch die Bedingung $g=-1$ verlangt wird.

Für einen im Anfangspunkt des Koordinatensystems befindlichen felderzeugenden Massenpunkt erhält man in erster Näherung die radialsymmetrische Lösung

(70) $\left\{\begin{array}{l}g_{\rho^{\sigma}}=-\delta_{\varrho \sigma}-\alpha^{\frac{x_{\rho} x_{\sigma}}{r^{3}}}(\varrho \text { und } \sigma \text { zwischen } 1 \text { und } 3) \\ g_{\rho^{4}}=g_{4^{\prime}}=0 \quad(\varrho \text { zwischen } 1 \text { und } 3) \\ g_{44}=1-\frac{\alpha}{r} .\end{array}\right.$ $\delta_{\varrho \sigma}$ ist dabei 1 bzw. 0 , je nachdem $\varrho=\sigma$ oder $\varrho \sigma, r$ ist die
Größe

$$
+\sqrt{x_{1}^{2}+x_{2}^{2}+x_{3}^{2}} \text {. }
$$

Dabei ist wegen $(68 \mathrm{a})$

$$
\alpha=\frac{x M}{4} \pi^{-}
$$

wenn mit $M$ die felderzeugende Masse bezeichnet wird. Daß durch diese Lösung die Feldgleichungen (außerhalb der Masse) in erster Näherung erfüllt werden, ist leicht $\mathrm{zu}$ verifizieren.

Wir untersuchen nun die Beeinflussung, welche die metrischen Eigenschaften des Raumes durch das Feld der Masse $M$ erfahren. Stets gilt zwischen den ,lokal“ (§ 4) gemessenen Längen und Zeiten $d s$ einerseits und den Koordinatendifferenzen $d x_{v}$ andererseits die Beziehung

$$
d s^{2}=g_{\mu \nu} d x_{\mu} d x_{\nu} .
$$

Für einen ,parallel“ der $x$-Achse gelegten Einheitsmaßstab wäre beispielsweise zu setzen

also

$$
d s^{2}=-1 ; d x_{2}=d x_{3}=d x_{4}=0,
$$

$$
-1=g_{11} d x_{1}{ }^{2} \text {. }
$$

Liegt der Einheitsmaßstab außerdem auf der $x$-Achse, so ergibt die erste der Gleichungen (70)

$$
g_{11}=-\left(1+\frac{\alpha}{r}\right) \text {. }
$$

Aus beiden Relationen folgt in erster Näherung genau

$$
d x=1-\frac{\alpha}{2 r} .
$$

Der Einheitsmaßstab erscheint also mit Bezug auf das Koordinatensystem in dem gefundenen Betrage durch das Vorhandensein des Gravitationsfeldes verkürzt, wenn er radial angelegt wird.

Analog erhält man seine Koordinatenlänge in tangentialer Richtung, indem man beispielsweise setzt

$d s^{2}=-1 ; d x_{1}=d x_{3}=d x_{4}=0 ; x_{1}=r, x_{2}=x_{3}=0$.

Es ergibt sich

$$
-1=g_{22} d x_{2}{ }^{2}=-d x_{2}^{2} .
$$

Bei tangentialer Stellung hat also das Gravitationsfeld des Massenpunktes keinen Einfluß auf die Stablänge.

Es gilt also die Euklidische Geometrie im Gravitationsfelde nicht einmal in erster Näherung, falls man einen und densel ben Stab unabhängig von seinem Ort und seiner Orientierung als Realisierung derselben Strecke auffassen will. Allerdings zeigt ein Blick auf (70a) und (69), daß die zu erwartenden Abweichungen viel zu gering sind, um sich bei der Vermessung der Erdoberfläche bemerkbar machen zu können. 


$$
-62-
$$

Es werde ferner die auf die Zeitkoordinate untersuchte Ganggeschwindigkeit einer Einheitsuhr untersucht, welche in einem statischen Gravitationsfelde ruhend angeordnet ist. Hier gilt für eine Uhrperiode

$$
d s=1 ; \quad d x_{1}=d x_{2}=d x_{3}=0 .
$$

oder

$$
\begin{gathered}
1=g_{44} d x_{4}{ }^{2} \\
d x_{4}=\frac{1}{\sqrt{y_{44}}}=\frac{1}{\sqrt{1+\left(y_{44}-1\right)}}=1-\frac{g_{44}-1}{2}
\end{gathered}
$$

$$
d x_{4}=1+\frac{x}{8 \pi} \int \frac{\varrho d \tau}{r} .
$$

Die Uhrr läuft also langsamer, wenn sie in der Nähe ponderabler Massen aufgestellt ist. Es folgt daraus, daß die Spektrallinien von der Oberfläche großer Sterne zu uns gelangenden Lichtes nach dem roten Spektralende verschoben erscheinen müssen. ${ }^{1}$ )

Wir untersuchen ferner den Gang der Lichtstrahlen im statischen Gravitationsfeld. Gemäß der speziellen Relativitätstheorie ist die Lichtgeschwindigkeit durch die Gleichung

$$
-d x_{1}^{2}-d x_{2}^{2}-d x_{3}^{2}+d x_{4}^{2}=0
$$

gegeben, also gemäß der allgemeinen Relativitätstheorie durch die Gleichung

$$
d s^{2}=g_{\mu \nu} d x_{\mu} d x_{v}=0 .
$$

Ist die Richtung, d. h. das Verhältnis $d x_{1}: d x_{2}: d x_{3}$ gegeben, so liefert die Gleichung (73) die Größen

$$
\frac{d x_{1}}{d x_{4}}, \quad \frac{d x_{2}}{d x_{4}}, \quad \frac{d x_{3}}{d x_{4}}
$$

und somit die Geschwindigkeit

$$
\sqrt{\left(\frac{d x_{1}}{d x_{4}}\right)^{2}+\left(\frac{d x_{2}}{d x_{4}}\right)^{2}+\left(\frac{d x_{3}}{d x_{4}}\right)^{2}}=\gamma \text {, }
$$

im Sinne der Euklidischen Geometrie definiert. Man erkennt leicht, daß die Lichtstrahlen gekrümmt verlaufen müssen mit

1) Für das Bestehen eines derartigen Effektes sprechen nach E. Freundlich spektrale Beobachtungen an Fixsternen bestimmter Typen. Eine endgültige Prüfung dieser Konsequenz steht indes noch aus.
$-63-$

Bezug auf das Koordinatensystem, falls die $g_{\mu \nu}$ nicht konstant sind. Ist $n$ eine Richtung senkrecht zur Lichtfortpflanzung, so ergibt das Huggenssche Prinzip, da $B$ der Lichtstrahl [in der Ebene $(\gamma, n)$ betrachtet] die Krïmmung - $d \gamma / \partial n$ besitzt.

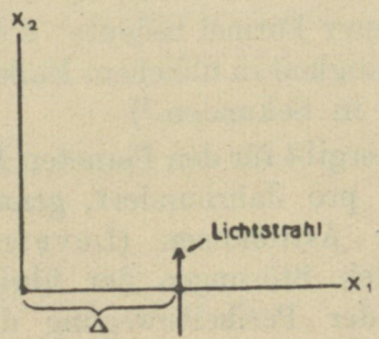

Wir untersuchen die Krümmung, welche ein Lichitstrahl leidet, der im Abstand $\Delta$ an einer Masse $M$ vorbeigeht. Wählt man das Koordinatensystem gemäß der vorstehenden Skizze, so ist die gesamte Biegung $B$ des Lichtstrahles (positiv gerechnet, wemn sie nach dem Ursprung hin konkav ist) in genügender Näherung gegeben durch

$$
B=\int_{-\infty}^{+\infty} \frac{\partial \gamma}{\partial x_{1}} d x_{2}
$$

während $(73)$ und $(70)$ ergeben

$$
\gamma=\sqrt{-\frac{g_{44}}{g_{22}}}=1+\frac{r}{2 r}\left(1+\frac{x_{2}^{2}}{r^{2}}\right) .
$$

Die Ausrechnung ergibt

$$
B=\frac{2 \alpha}{\Delta}=\frac{x M}{2 \pi \Delta} .
$$

Ein an der Sonne vorbeigehender Lichtstrahl erfährt demnach eine Biegung von 1,7", ein am Planeten Jupiter vorbeigehender eine solche von etwa $0,02^{\prime \prime}$.

Berechnet man das Gravitationsfeld um eine Größenordnung genauer, und ebenso mit entsprechender Genauigkeit die Bahnbewegung eines materiellen Punktes von relativ unendlich kleiner Masse, so erhält man gegenüber den KeplerNewtonschen Gesetzen der Planetenbewegung eine Abweichung von folgender Art. Die Bahnellipse eines Planeten er- 
fährt in Richtung der Bahnbewegung eine langsame Drehung vom Betrage

(75)

$$
\varepsilon=24 \pi^{3} \frac{a^{2}}{T^{2} c^{2}\left(1-e^{2}\right)}
$$

pro Umlauf. In dieser Formel bedeutet $a$ die große Halbachse, $c$ die Lichtgeschwindigkeit in üblichem $T$ die Umlaufszeit in Sekunden. ${ }^{1}$ )

Die Rechnung ergibt für den Planeten Merkur ejne Drehung der Bahn um 43" pro Jahrhundert, genau entsprechend der Konstatierung der Astronomen (Leverrier); diese fanden nämlich einen durch Störungen der übrigen Planeten nicht erklärbaren Rest der Perihelbewegung dieses Planeten von der angegebenen Größe.

1) Bezüglich der Rechnung verweise ich auf die Originalabhandlungen A. Einstein, Sitzungsber. d. PreuB. Akad, d. Wiss. 47. p. 831. 1915. - K. Schwarzschild, Sitzungsber. d. Preuß. Akad. d. Wiss. 7. p. 189. 1916. 